\title{
Research on Slicing Chip Forming and Wear Analysis of Arc-tooth Slice Tool With Equal-rake Angle
}

\section{Xiaoqiang Wu}

Key Laboratory of Mechanism and Equipment Design of Ministry of Education, Tianjin University, Tianjin, 300072, China

YONGQUAN JIN ( $\nabla_{\text {tju_jin@163.com ) }}$

Tianjin University

\section{Research Article}

Keywords: Equal rake angle, Arc-tooth slice tool, Chip, Load, Wear

Posted Date: May 27th, 2021

DOI: https://doi.org/10.21203/rs.3.rs-535415/v1

License: (c) (i) This work is licensed under a Creative Commons Attribution 4.0 International License.

Read Full License 


\title{
Research on slicing chip forming and wear analysis of arc-tooth
}

\section{slice tool with equal-rake angle}

\author{
Xiaoqiang $\mathrm{Wu}^{1,2}$, Yongquan $\mathrm{Jin}^{1 *}$ \\ 1. Key Laboratory of Mechanism and Equipment Design of Ministry of Education, Tianjin \\ University, Tianjin, 300072, China \\ 2. College of Mechanical Engineering, Inner Mongolia University for the Nationalities, Tongliao \\ 028000, China \\ Corresponding author: 1. Yongquan Jin, Key Laboratory of Mechanism and Equipment Design of \\ Ministry of Education, Tianjin University, Tianjin, 300072, China \\ 2. Xiaoqiang Wu, Key Laboratory of Mechanism and Equipment Design of Ministry of Education, \\ Tianjin University, Tianjin, 300072, China \\ Tel.: +86-022-87880066 \\ Fax: $+86-022-27401624$ \\ E-mail: tju_jin@163.com \\ wangzai8402@163.com
}

\begin{abstract}
As a new technology of gear processing, the gear slicing technology has distinct characteristics and broad application prospects. However, the lack in wear and life of the slice tool hinders the further widespread application of the gear slicing technology. Considering the chip forming mechanism is an important basis affecting the wear and service life of the cutter, this paper takes the arc-tooth slice tool with equalrake angle (ATST-ERA) as the carrier and studies the chip forming and tool wear. Aiming at the particularity of the slicing process, the cutting process is unified and abstracted, and the cutting edge model, the cutting edge race model and the workpiece tooth surface model of the ATST-ERA are established, and then the undeformed chip geometry model is obtained. The correctness of the parametric model of the undeformed chip was verified by comparative analysis. Based on this model, the load of the cutting edge is calculated using the Kienzle empirical formula of the cutting force. By analyzing the relationship with the wear of the rake face, the positive correlation between the cutting edge load and the rake face wear of the ATST-ERA is found. The chip forming modeling method proposed in this paper provides a theoretical basis for the control of the slicing chip forming and the improvement of tool wear.
\end{abstract}

Key words: Equal rake angle, Arc-tooth slice tool, Chip, Load, Wear 


\section{Introduction}

As a totally new gear machining technology, the gear slicing technology is not realized until the 21 st century. The gear slicing technology can not only complete the machining of special structural gears such as thin-walled and non-penetrating internal helical teeth, but also has the characteristics of high efficiency, high precision and environmentally friendly. Therefore, many scholars have studied the gear slicing technology ${ }^{[1-12]}$. Though scholars have achieved breakthrough, the gear slicing technology has not been widely used. The reason is mainly because the slice tool is easy to wear and has a short service life. According to the metal cutting principle, the chip forming law is an important factor affecting tool wear. Because of this, in order to improve the wear performance of the slice tool and improve its service life, it is necessary to conduct an in-depth study on the chip forming mechanism during the slicing process.

Schulze et al. analyzed the chip forming mechanism and its effect on machining reliability by modeling the motion process of the gear skiving ${ }^{[13]}$. Mazak et al. used the simulation method to study the machining process of the intermittent cutting of the bevel gear. It was found that the peak point of the chip thickness could not be explained from the physical and kinematics, by analyzing the chip shape. Therefore, the method of calculating the chip thickness was proposed to describe the thickness of each part of the chip ${ }^{[14]}$. Tapoglou established a chip simulation model for power skiving in the CAD simulation environment. Based on this model, the geometry and size of nondeformed chips can be obtained ${ }^{[15]}$. Pierce et al. used a solid modeling software to create a virtual model of the power skiving process, extracting the chip geometry ${ }^{[16]}$.

The above research is mainly based on simulation techniques, and the relevant research results have not been experimentally verified. In order to carry out in-depth research on the chip forming mechanism during slicing process, we can learn from the related research of chip forming of other machining process, such as turning, milling and hobbing.

Uysa, Su and Zang used the cutting process of stainless steel, graphite cast iron and titanium alloy as the carrier, and studied the chip forming mechanism and threedimensional shape of the chip by means of finite element simulation and cutting experiments. Then they analyzed the effects of cutting speed, feed rate and cutting temperature on the chip shape, and calculated the chip thickness, chip curl radius, shear zone thickness, etc. according to the chip model ${ }^{[17-19]}$. Ahmed et al. considered the 
influence of tool wear and established the chip model of AISI 304 turning in high pressure cooling environment. It was verified that the model prediction value of chip curl radius was basically consistent with the measured value ${ }^{[20]}$. Rakesh et al. studied the influence of cutting speed on chip shape by using dry-cutting chrome-nickel alloy as a carrier, and verified it by experiments ${ }^{[21]}$. Molnar et al. used orthogonal cutting as the research object. By comparing non-stationary cutting and stationary cutting experiments, the influence of cutting thickness on cutting force was analyzed, and the theoretical model related to chip breaking was evaluated ${ }^{[22]}$.

Dou et al. established a kinematic transcendental equation that satisfies the conditions of milling chip formation. The thickness of the milling chip was obtained by numerical and approximate analytical methods. The theoretical model and the superiority of its solution was verified by comparing with the existing model and its computational efficiency. ${ }^{[23]}$. Nikawa et al. used CAM simulation software to control the chip thickness by modifying the cutting parameters of the ball-end milling cutter in the NC code, and finally improved the machining ability of the machining method ${ }^{[24]}$.

Harry et al. proposed a theoretical modeling method for the machining process of turning and milling to predict the geometry of the uncut chip, and then calculated the cutting force, and verified the accuracy of the cutting force calculation result ${ }^{[25]}$. Berenji et al. considered the turning and milling process under different machining conditions, studied the influence of machining process parameters on the chip forming mechanism. It was verified that good process parameters improved the machining quality ${ }^{[26]}$. Bergmann et al. considered the limitations of specific machining methods and specific workpiece materials, and studied the chip forming mechanism during the cutting process of circular cutting edges with different microscopic shapes, and verified them by planning and milling experiments ${ }^{[27]}$

Chen et al. used the gear hobbing as the carrier to establish the space forming model of cylindrical gear of multi-edge interrupted cutting. The chip geometry was obtained by numerical method, and the relationship between the load distribution and wear of the cutting edge was further analyzed ${ }^{[28,29]}$. Likely, the gear hobbing process is the object. Li et al. used the finite element software to establish the simulation model of gear hobbing of high-speed and dry-cutting, and obtained the deformation law of the chip during the gear hobbing process and the wear state of the hob. The model was validated by the actual gear hobbing experiment ${ }^{[30]}$. Zhang used the solid modeling method to realize the material removal simulation of the gear hobbing process, and 
obtained the geometric entity of the undeformed chip. But the research result was not verified by experiments ${ }^{[31]}$.

As can be seen from the above literature, the motion relationship involved in the gear hobbing process is more complicated than in turning and milling process. In order to study the chips in the gear hobbing process, it is necessary to analyze and simplify the geometry of the gear hobbing process, and to study the chips of the gear hobbing process based on the orthogonal or bevel cutting principle. Similarly, this paper draws on the research method of chips in cutting process such as turning, milling and hobbing, analyzes the machining process of the ATST-ERA, and establishes the parametric model of the gear slicing process and the geometry model of the undeformed chip. The geometry model of the undeformed chip is verified by comparison with the chips obtained from the gear slicing experiment. On this basis, the cutting edge load is calculated based on the empirical formula. By comparing the load distribution of the cutting edge with the wear of the rake face, the positive correlation between the load distribution of the cutting edge and the wear of the rake face is obtained, which further verifies the correctness of the theoretical model established in this paper.

\section{Mathematics expression of cutting process}

\subsection{The unified and abstract process of slicing}

The gear slicing technology can realize the machining of various gears such as internal teeth, external teeth, straight teeth and helical teeth. Regardless of the type of gear machining, the slicing process is based on the conjugate rotary cutting principle. The gear slicing process is similar to the gear meshing process. The tool and the workpiece rotate in a certain speed ratio. The cutting edge that meets the conjugate condition of the line and surface continuously removes excess material during the continuous sweeping process, and finally forms the tooth surface of the workpiece. According to the principle of conjugate rotary cutting, there are three kinds of motions involved in the gear slicing process, including the rotation of the workpiece, the rotation of the slice tool and the feed of the workpiece or slice tool along the axial direction. In addition, a shaft angle is always maintained between the tool axis and the workpiece axis during the movement of the tool and the workpiece. For the machining of different types of gears, the biggest difference lies in the relative position of the tool and the workpiece, and the cutting amount.

Looking at the tool axis as a reference, if the tool rotates counterclockwise with 
respect to the workpiece axis, it is considered to be "left pendulum"; if the tool rotates clockwise with respect to the workpiece axis It is considered to be "right pendulum". When the workpiece rotates counterclockwise with respect to the workpiece axis, the angular velocity is positive, and vice versa; when the tool rotates counterclockwise with respect to the tool axis, the angular velocity is positive, and vice versa.

As shown in Figure 1, when slicing a spur and internal gear, if the cutter shaft swings to the left, the cutter rotates counterclockwise around the tool axis, and the workpiece rotates counterclockwise around the workpiece axis. If the cutter shaft swings to the right, the cutter rotates clockwise around the tool axis, and the workpiece rotates clockwise around the workpiece axis. As shown in Figure 2, when cutting a spur and external gear, if the cutter shaft swings to the left, the cutter rotates counterclockwise around the tool axis, and the workpiece rotates clockwise around the workpiece axis. If the cutter shaft swings to the right, the cutter rotates clockwise around the tool axis, and the workpiece rotates counterclockwise about the workpiece axis. Considering the shaft angle between the tool axis and the workpiece axis, the helix angle of the slice tool must be equal to shaft angle to ensure that the tooth direction of the cutter is consistent with the direction of the tooth groove of the workpiece, so that the tooth groove of the spur gear can be machined.

For the cutting of the spur gear, the difference between the machining of the internal gear workpiece and the external gear workpiece is the center distance value and the tool axis direction, as shown in Figure 3. According to the basic principle of the above-mentioned caries processing, the caries processing process of different types of gears is simplified into a unified motion abstract model, as shown in Figure 4.

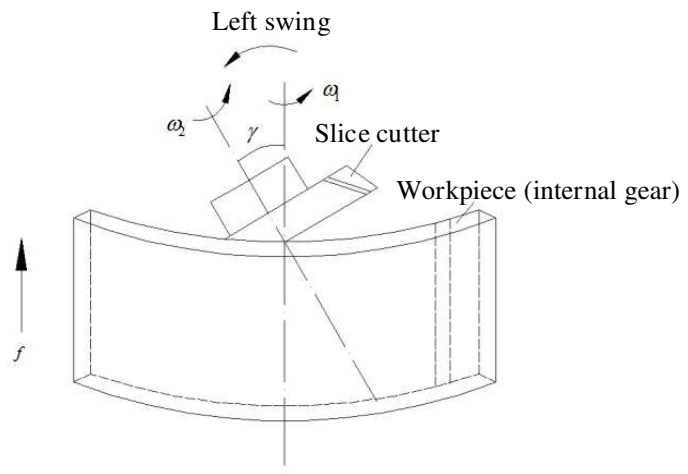

(a)

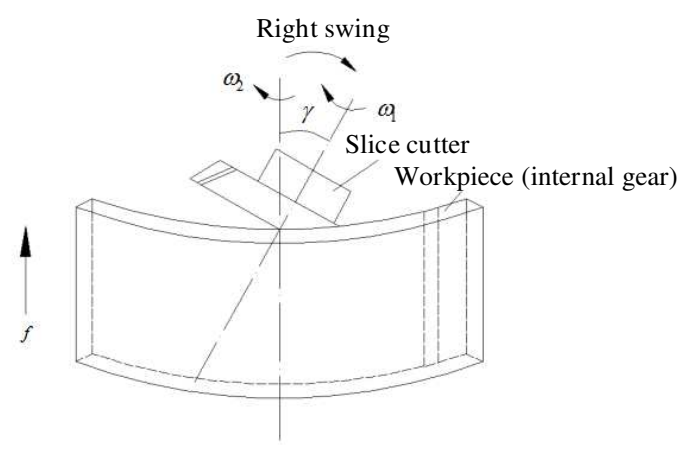

(b)

Figure 1 Slicing of the spur and internal gear 


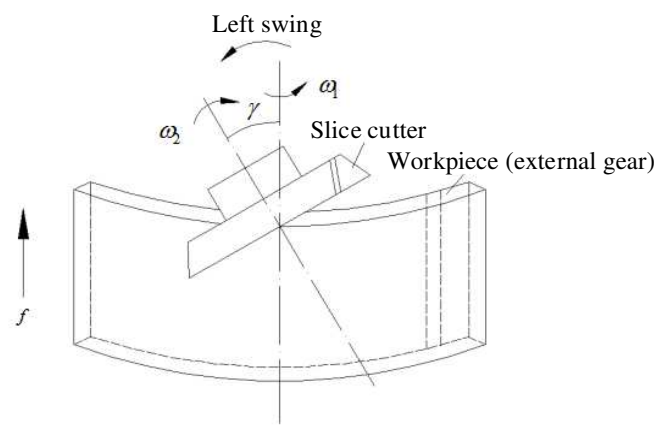

(a)

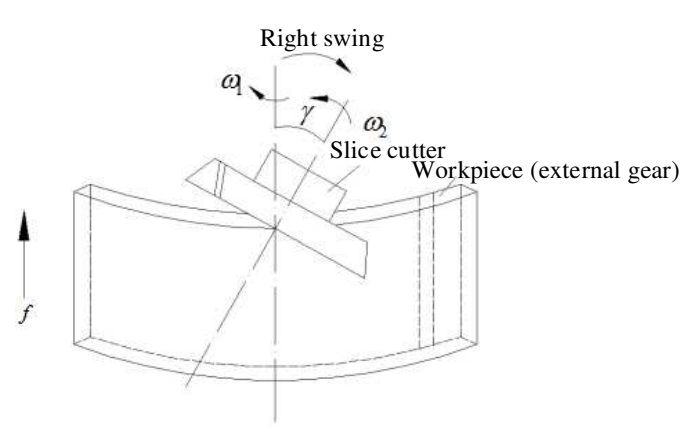

(b)

Figure 2 Slicing of the spur and external gear

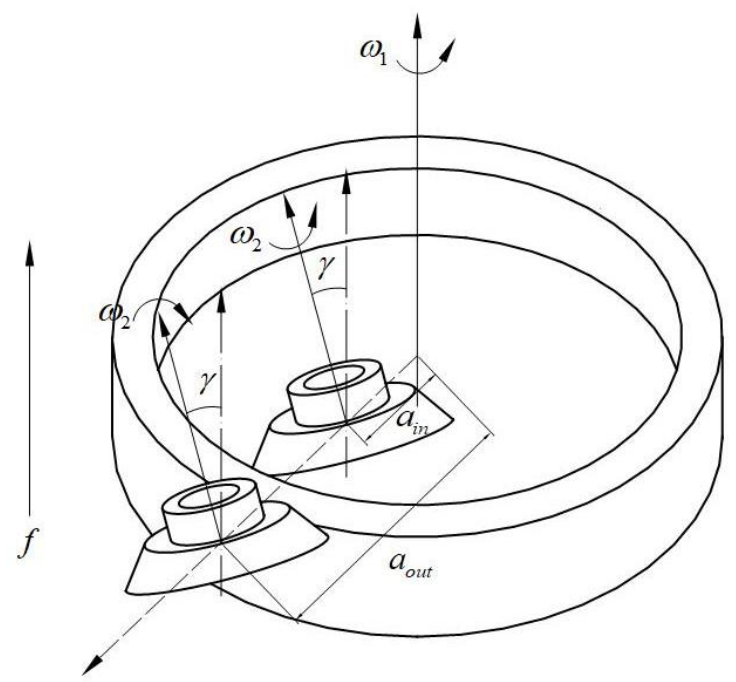

Figure 3 Similarities and differences between the slicing of internal and external gears

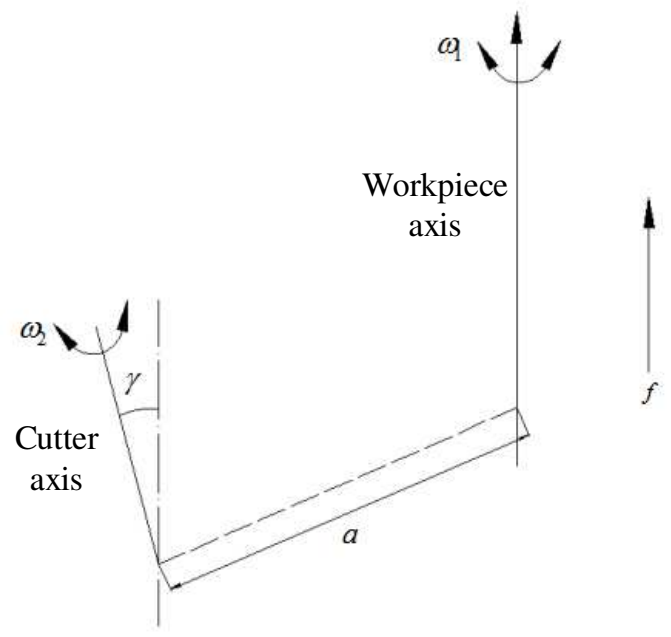

Figure 4 Motion abstract model of gear slicing 
According to the position and motion relationship between the tool and the workpiece, the coordinate system of slicing is established, as shown in Figure 5. In the figure, the coordinate system $S_{1}\left(o_{1}, x_{1}, y_{1}, z_{1}\right)$ is the workpiece coordinate system, $o_{1}$ represents the coordinate origin of the $S_{1}$ coordinate system, the unit vectors of $x_{1} 、 y_{1}$ 、 $z_{1}$ are $i_{1} 、 j_{1} 、 k_{1}$, respectively; and the coordinate system $S_{2}\left(o_{2}, x_{2}, y_{2}, z_{2}\right)$ is the tool coordinate system, $o_{2}$ is the coordinate origin of the $S_{2}$ coordinate system, and the unit vectors of $x_{2} 、 y_{2} 、 z_{2}$ are $i_{2} 、 j_{2} 、 k_{2}$, respectively; the coordinate system $S_{p}\left(o_{p}, x_{p}, y_{p}\right.$, $z_{p}$ ) is The auxiliary coordinate system of the coordinate system $S_{1}, o_{p}$ represents the coordinate origin of the $S_{p}$ coordinate system, the unit vectors of $x_{p} 、 y_{p} 、 z_{p}$ are $i_{p} 、 j_{p}$ 、 $k_{p}$, respectively; the coordinate system $S_{0}(o, x, y, z)$ is the auxiliary coordinate system of $S_{2}, o$ represents the coordinate origin of the $S_{0}$ coordinate system, and the unit vectors of $x, y, z$ are respectively $i 、 j 、 k$. The coordinate system $S_{1}$ is used to establish the workpiece tooth surface model, and the coordinate system $S_{2}$ is used to establish the tool model. The spatial positions of the auxiliary coordinate systems $S_{p}$ and $S_{0}$ are fixed and do not change with time. The center distance $a$ represents the vertical distance between the tool axis and the workpiece axis, and the axis angle $\gamma$ represents the angle between the tool axis and the workpiece axis. $\varphi_{1}$ represents the angle of the workpiece coordinate system $S_{1}$ as the workpiece rotates relative to the initial position, and $l$ represents the distance that the workpiece coordinate system $S_{1}$ moves relative to the auxiliary coordinate $S_{p}$ along the workpiece axis (the positive direction of the $z_{p}$ axis), and $\varphi_{2}$ represents the angle of the tool coordinate system $S_{2}$ as the tool rotates relative to the initial position.

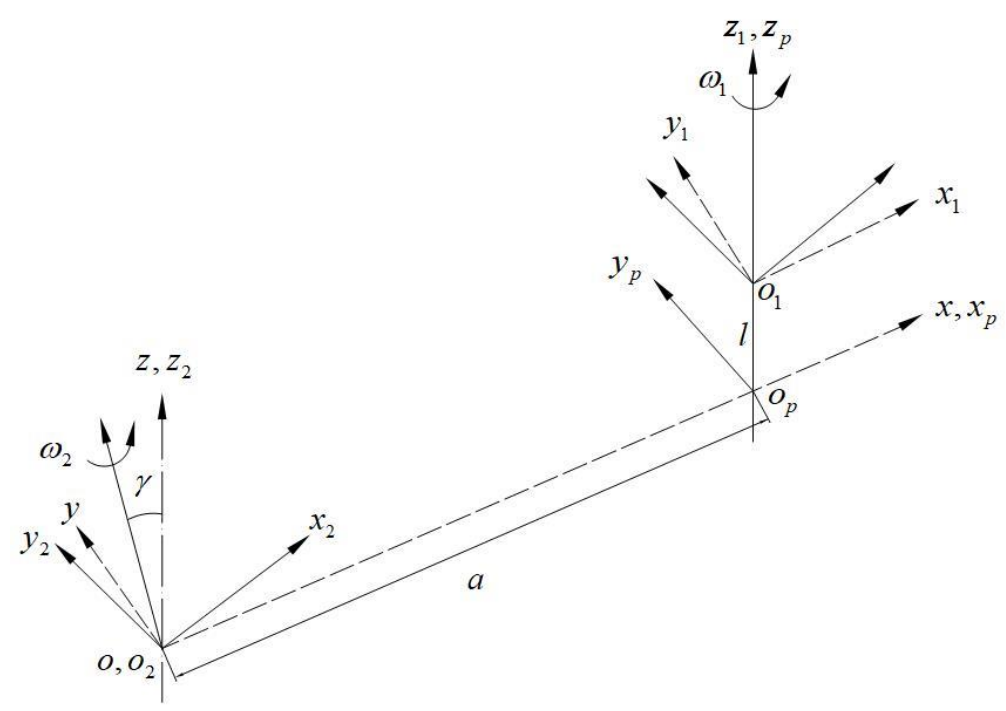


According to the gear machining coordinate system shown in Figure 5, the total transformation matrix of the tool coordinate system $S_{2}$ to the workpiece coordinate system $S_{1}$ is:

$$
\boldsymbol{T}=\boldsymbol{T}_{20} \cdot \boldsymbol{T}_{0 p} \cdot \boldsymbol{T}_{p 1}
$$

In this formula

$$
\begin{aligned}
& \boldsymbol{T}_{20}=\left[\begin{array}{cccc}
\cos \varphi_{1} & -\sin \varphi_{1} & 0 & 0 \\
\sin \varphi_{1} & \cos \varphi_{1} & 0 & 0 \\
0 & 0 & 1 & l \\
0 & 0 & 0 & 1
\end{array}\right] \\
& \boldsymbol{T}_{0 p}=\left[\begin{array}{cccc}
1 & 0 & 0 & a \\
0 & \cos \gamma & -\sin \gamma & 0 \\
0 & \sin \gamma & \cos \gamma & 0 \\
0 & 0 & 0 & 1
\end{array}\right] \\
& \boldsymbol{T}_{p 1}=\left[\begin{array}{cccc}
\cos \varphi_{2} & \sin \varphi_{2} & 0 & 0 \\
-\sin \varphi_{2} & \cos \varphi_{2} & 0 & 0 \\
0 & 0 & 1 & 0 \\
0 & 0 & 0 & 1
\end{array}\right]
\end{aligned}
$$

In the above formula, $\boldsymbol{T}_{20}$ represents a transformation matrix of the tool coordinate system $S_{2}$ to the auxiliary coordinate system $S_{0}, \boldsymbol{T}_{0 p}$ represents a transformation matrix of the auxiliary coordinate system $S_{0}$ to the auxiliary coordinate system $S_{p}$, and $\boldsymbol{T}_{p 1}$ represents a transformation matrix of the auxiliary coordinate system $S_{p}$ to the workpiece coordinate system $S_{1}$.

\subsection{Model of edge-sweeping surface}

According to the conjugate rotary cutting principle, the cutting edge and the theoretical tooth surface of the workpiece satisfy the conjugate relationship. Since many curves on the conjugate surface of the tooth surface of the workpiece satisfy the conjugate relationship with the tooth surface of the workpiece, a curve that satisfies the conjugate relationship with the tooth surface of the workpiece from tooth root to tooth crest is selected as the cutting edge of the slice tool. Firstly, the conjugate surface of the theoretical tooth surface of the workpiece is obtained, and then a plane or other regular curved surface is used to intersect with the conjugate surface, and the intersection line 
is the cutting edge curve. The method of deriving the cutting edge curve is basically the same as that used by Wang et al. ${ }^{[12]}$. It will not be repeated here. The cutting edge curve of the slice tool is shown in Figure 6.

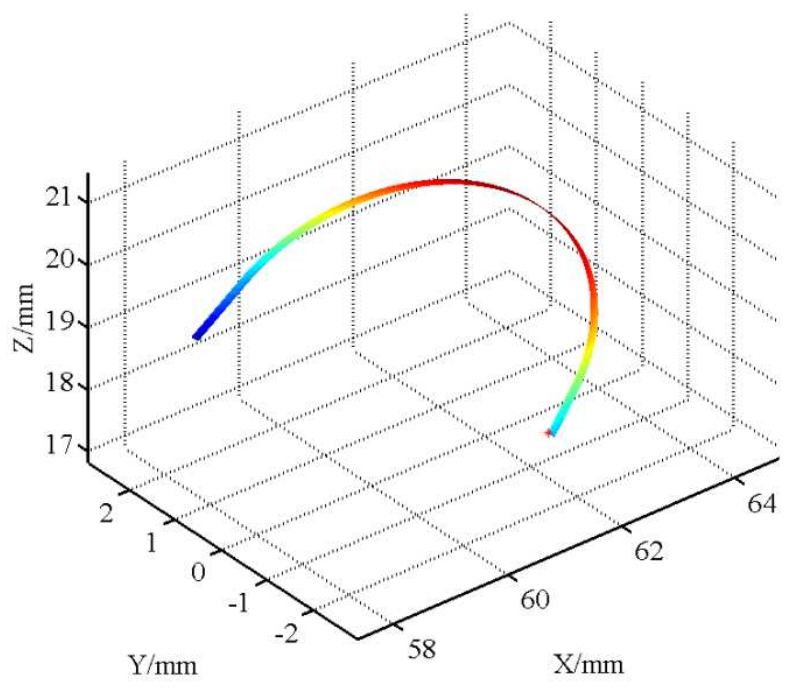

图 6 The cutting edge of the ATST-ERA

The final formed tooth surface of the workpiece is made by multiple cuts of the cutting edge of the cutter, and the motion trace of the cutting edge relative to the tooth surface of the workpiece is the edge-sweeping surface. The cutting edge of the slice tool is usually indicated on the tool coordinate system $S_{2}$. The tooth surface of the workpiece is usually indicated on the workpiece coordinate system $S_{1}$. To accurately calculate the position relationship between the edge-sweeping surface and the tooth surface of the workpiece, the coordinate transformation is necessary to be done between coordinate system $S_{2}$ and $S_{1}$. In order to facilitate the expression later, the cutting edge on the tool coordinate system $S_{2}$ is selected to be transformed to the workpiece coordinate system $S_{1}$. On the workpiece coordinate system $S_{1}$, the tooth surface of the workpiece is fixed. The cutting edge is fixed on the tool coordinate system $S_{2}$. After the cutting edge on the tool coordinate system $S_{2}$ is transformed to the workpiece coordinate system $S_{1}$, the position of the cutting edge changes with time, and a motion trajectory is formed on the workpiece coordinate system $S_{1}$, which is the edge-sweeping surface.

The spatial curved surface formed by the cutting edge of any one of the arc tooth cutters swept on the workpiece coordinate system $S_{1}$ is shown in Figure 7. In the figure, $D_{1}$ represents the cutting edge at time $t_{1}, D_{i}$ represents the cutting edge at a certain time 
$t_{i}$ in $\left[t_{1}, t_{2}\right]$, and $D_{n}$ represents the cutting edge at time $t_{2}$.

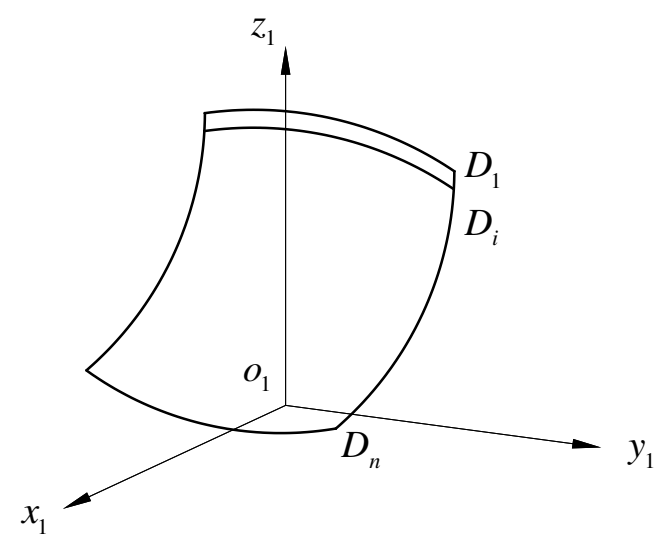

Figure 7 Edge-sweeping surface formed by the cutting edge on the workpiece coordinate system $S_{1}$

Based on the space kinematics principle, the equation of the edge-sweeping surface can be obtained as

$$
\boldsymbol{r}_{j}(r, \varphi)=\boldsymbol{T} \cdot \boldsymbol{r}_{r}(r)
$$

where, $\boldsymbol{r}_{j}$ represents the vector of the point on the $j$-th edge-sweeping surface on the workpiece coordinate system $S_{1}, \boldsymbol{r}_{\mathrm{r}}$ represents the vector of the point on the cutting edge on the tool coordinate system $S_{2}, j$ indicating the distance between the point on the cutting edge of the $j$-th edge-sweeping surface on the tool coordinate system $S_{2}$ and the tool axis, $\boldsymbol{T}$ represents the total transformation matrix from the tool coordinate system $S_{2}$ to the workpiece coordinate system $S_{1}$. Let

$$
\begin{aligned}
\boldsymbol{r}_{j}(r, \varphi) & =\left[\begin{array}{llll}
x_{x 1} & y_{x 1} & z_{x 1} & 1
\end{array}\right]^{\prime} \\
\boldsymbol{r}_{\mathrm{r}}(r) & =\left[\begin{array}{llll}
x_{2} & y_{2} & z_{2} & 1
\end{array}\right]^{\prime}
\end{aligned}
$$

The simultaneous equations (1), (5), (6), (7) can obtain the parametric equation of the edge-sweeping surface as

$$
\left[\begin{array}{c}
x_{x 1} \\
y_{x 1} \\
z_{x 1} \\
1
\end{array}\right]=\left[\begin{array}{c}
x_{2} A+y_{2} B+z_{2} C-a \cos \varphi_{1} \\
x_{2} D+y_{2} E+z_{2} F+a \sin \varphi_{1} \\
x_{2} \sin \varphi_{2} \sin \gamma+y_{2} \cos \varphi_{2} \sin \gamma+z_{2} \cos \gamma-l \\
1
\end{array}\right]
$$

where, $A=\cos \varphi_{1} \cos \varphi_{2}+\sin \varphi_{1} \sin \varphi_{2} \cos \gamma, B=-\cos \varphi_{1} \sin \varphi_{2}+\sin \varphi_{1} \cos \varphi_{2} \cos \gamma$, $C=-\sin \varphi_{1} \sin \gamma, \quad D=-\sin \varphi_{1} \cos \varphi_{2}+\cos \varphi_{1} \sin \varphi_{2} \cos \gamma$, $E=\sin \varphi_{1} \sin \varphi_{2}+\cos \varphi_{1} \cos \varphi_{2} \cos \gamma, F=-\cos \varphi_{1} \sin \gamma \cdot x_{2} 、 y_{2} 、 z_{2}$ respectively represent 
components of the vector of the points on the cutting edge in the tool coordinate system $\mathrm{S}_{2}$ on the $x_{2}$ axis, the $y_{2}$ axis, and the $z_{2}$ axis, all of which are functions of $r$. Analysis of equations (5) and (8) shows that the mathematical model of the edge-sweeping surface can be seen as a parametric equation for the variables $r$ and $\varphi$. As shown in Figure 8, the edge-sweeping surface of the arc-tooth slice tool is obtained according to the above formula.

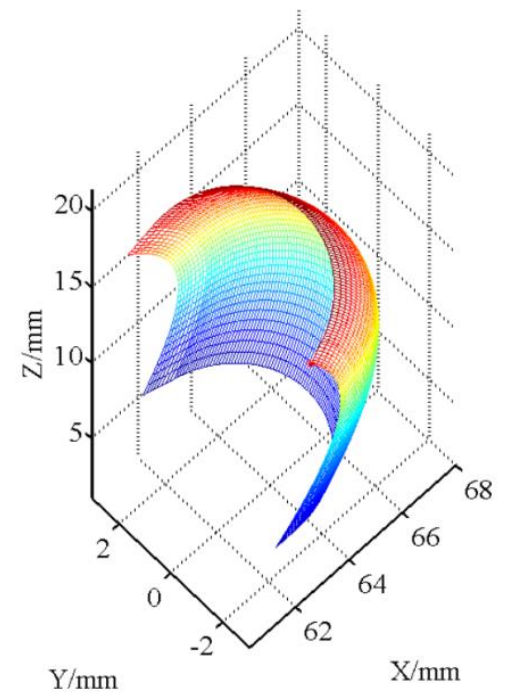

Figure 8 Edge-sweeping surface of the ATST-ERA

2.3 Model of edge-sweeping surface family

In the slicing process, the final tooth surface of the workpiece is obtained after sweeping by multiple edge-sweeping surfaces. Take a certain tooth slot $E_{i}$ of the workpiece as an example. Assume that one of the cutter teeth $F_{i}$ firstly contacts and cuts the slot $E_{i}$. After the workpiece rotates one revolution, the other cutter tooth $F_{j}$ of the slice tool continues to contact and cut the slot $E_{i}$. If the ratio of the number of teeth of the workpiece to the number of teeth of the slice tool is an integer, $F_{i}=F_{j}$, and if the number of teeth of the workpiece is equal to the number of teeth of the slice tool, $F_{i} \neq F_{j}$. Assuming that the time span of the edge-sweeping surface $\Sigma_{1}$ slicing the tooth slot $E_{i}$ is $\left[t_{1}, t_{2}\right]$. The edge-sweeping surface $\Sigma_{1}$ is formed by the cutting edge of the slice tool $F_{i}$ relative to of the workpiece tooth groove $E_{i}$. Then 


$$
\left\{\begin{array}{l}
t_{1}^{\prime}=t_{1}+\frac{2 \pi}{\omega_{1}} \\
t_{2}^{\prime}=t_{2}+\frac{2 \pi}{\omega_{1}}
\end{array}\right.
$$

After the workpiece is rotated for $n$ weeks, the time span of the edge-sweeping surface $\Sigma_{n}$ slicing the tooth slot $E_{i}$ is $\left[t_{1}, t_{2}\right]$. Then

$$
\left\{\begin{array}{l}
t_{1}^{n}=t_{1}+\frac{2 \pi(\mathrm{n}-1)}{\omega_{1}} \\
t_{2}^{n}=t_{2}+\frac{2 \pi(\mathrm{n}-1)}{\omega_{1}}
\end{array}\right.
$$

The edge-sweeping surfaces $\left(\Sigma_{1}, \Sigma_{2}, \cdots, \Sigma_{n}, n \geq 3\right)$ formed on the tooth groove $E_{i}$ is the edge-sweeping surface family. Assume that the center angle of the tooth $F_{i}$ on the slice tool is $\theta$ at the time $t_{1}$. The center angle of the tooth $F_{k}$ on the slice tool is $\theta_{\mathrm{k}}=\theta+\Delta \theta$ after the workpiece is rotated for $n$ weeks. Where

$$
\Delta \theta=\frac{2 \pi(n-1)\left(z_{1}-z_{2}\right)}{z_{2}}
$$

The simultaneous formulae (6) and (11) can obtain the parametric equation of the cutting edge on the tooth $F_{k}$

$$
\boldsymbol{r}_{\mathrm{r}}(r, k)=\left[\begin{array}{llll}
x_{2 \mathrm{k}} & y_{2 \mathrm{k}} & z_{2 \mathrm{k}} & 1
\end{array}\right]
$$

According to the formulas (1), (11) and (12), the parametric equation of the edgesweeping surface family can be obtained as

$$
\left[\begin{array}{c}
x_{\mathrm{xk}} \\
y_{\mathrm{xk}} \\
z_{\mathrm{xk}} \\
1
\end{array}\right]=\left[\begin{array}{c}
x_{2 \mathrm{k}} A_{\mathrm{k}}+y_{2 \mathrm{k}} B_{\mathrm{k}}+z_{2 \mathrm{k}} C_{\mathrm{k}}-a \cos \theta_{1 \mathrm{k}} \\
x_{2 \mathrm{k}} D_{\mathrm{k}}+y_{2 \mathrm{k}} E_{\mathrm{k}}+z_{2 \mathrm{k}} F_{\mathrm{k}}+a \sin \theta_{1 \mathrm{k}} \\
x_{2 \mathrm{k}} \sin \theta_{2 \mathrm{k}} \sin \alpha+y_{2 \mathrm{k}} \cos \theta_{2 \mathrm{k}} \sin \alpha+z_{2 \mathrm{k}} \cos \alpha-l \\
1
\end{array}\right]
$$

where, $A_{\mathrm{k}}=\cos \theta_{1 \mathrm{k}} \cos \theta_{2 \mathrm{k}}+\sin \theta_{1 \mathrm{k}} \sin \theta_{2 \mathrm{k}} \cos \alpha$,

$B_{\mathrm{k}}=-\cos \theta_{1 \mathrm{k}} \sin \theta_{2 \mathrm{k}}+\sin \theta_{1 \mathrm{k}} \cos \theta_{2 \mathrm{k}} \cos \alpha, C_{\mathrm{k}}=-\sin \varphi_{1 \mathrm{k}} \sin \alpha$, $D_{\mathrm{k}}=-\sin \theta_{1 \mathrm{k}} \cos \theta_{2 \mathrm{k}}+\cos \theta_{1 \mathrm{k}} \sin \theta_{2 \mathrm{k}} \cos \alpha, \quad E_{\mathrm{k}}=\sin \theta_{1 \mathrm{k}} \sin \theta_{2 \mathrm{k}}+\cos \theta_{1 \mathrm{k}} \cos \theta_{2 \mathrm{k}} \cos \alpha$, $F_{\mathrm{k}}=-\cos \theta_{1 \mathrm{k}} \sin \alpha$. The edge-sweeping surface family of the arc-tooth slice tool is shown in Figure 9. 


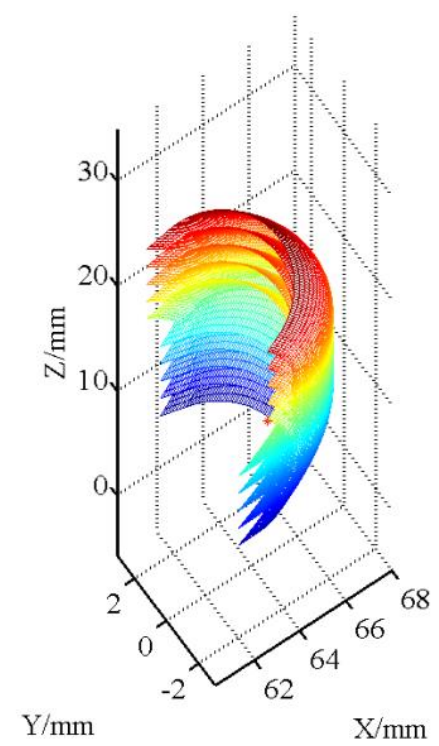

Figure 9 The edge-sweeping surface family of a cutting edge

\subsection{Model of workpiece tooth surface}

The cutting amount of the caries processing is very small, and it takes many times to complete the machining of the tooth surface. In order to make the cutting amount as reasonable as possible, the cutting layer in the radial direction is divided into multiple parts, and the layer-by-layer cutting of the workpiece in the radial direction is realized by sequentially adjusting the vertical distance (center distance) between the tool axis and the workpiece axis, until the workpiece tooth surface meets the accuracy requirements.

The slicing of the tooth groove $E_{i}$ is taken as an example. Figure 10 is a schematic diagram of the sweeping of the tooth slot $E_{i}$ by two adjacent edge-sweeping surfaces. It is assumed that the center distance between the arc-tooth slice cutter and the workpiece is $a_{1}$. The edge-sweeping surface $\Sigma_{1}$ can only remove part material of the tooth groove $E_{i}$. The edge-sweeping surface is formed by the cutter tooth $F_{i}$ of the arctooth slice cutter relative to the tooth slot $E_{i}$ of the workpiece. When the edge-sweeping surface family $\left(\Sigma_{1}, \Sigma_{2}, \cdots, \Sigma_{n}\right), n \geq 3$ all cutting the tooth slot $E_{i}$, the arc-tooth slice cutter completes the machining of the tooth slot $E_{i}$, and at the same time, it completes the processing of all the tooth slot of the workpiece. Assuming that the cutting amount is $\delta_{1}$, the total cutting amount $\delta$ needs to be completed to form the tooth surface of the workpiece that meets the accuracy requirements. Adjust the center distance between the slice tool and the workpiece so that the center distance is $a_{2}$. Repeat the previous process and the cutting amount that can be completed is $\delta_{2}$. Cycling and reciprocating, when 
the cumulative amount of cutting reaches $\delta$, the arc-tooth slice cutter completes the machining of the tooth surface of the workpiece to form the final gear. The method of deriving the tooth surface equation is basically the same as that used by Wang et al. ${ }^{[12]}$. It will not be repeated here. The tooth surface of the arc-tooth gear is shown in Figure 11.

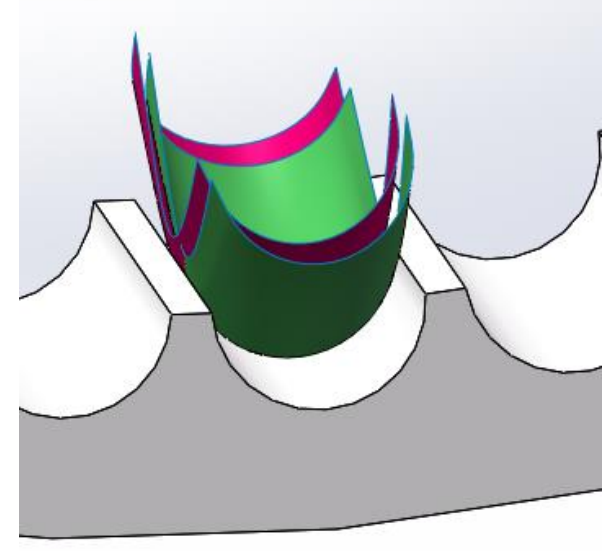

Figure 10 Schematic diagram of the tooth surface of the workpiece swept by the adjacent edge-sweeping surface

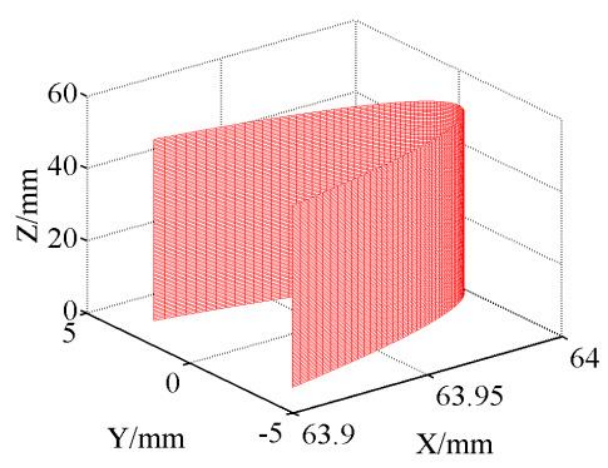

Figure 11 Tooth surface of arc-tooth gear

\section{Geometry model of undeformed chip and its verification}

\subsection{Geometry model of undeformed chip}

When the arc-tooth slice cutter is cutting the gear workpiece, the cutting thickness corresponding to the different positions of the cutting edge on the cutter tooth is uneven and changes with time, and the material remove by each cutting edge presents a complicated geometry, that is, chips. In engineering practice, there are multiple forces 
between the tool and the workpiece during the generation of the chip, and the chips generated during the slicing process are all deformed chips. In order to simplify the model, the other forces received during the chip forming process are ignored, and the undeformed chips in the slicing are mainly studied.

For the gear slicing process, a certain time period $\left[t_{1}, t_{2}\right]$ is selected, where $t_{1}$ represents the start time of the edge-sweeping surface and $t_{2}$ represents the end time of the edge-sweeping surface. As shown in Figure 12, the edge-sweeping surface is divided into two stages of sweeping in and sweeping out with the lowest point in the middle of the blade defined as the dividing line. The time corresponding to the dividing line is $\left(t_{2}-t_{1}\right) / 2$, corresponding to the sweeping phase. The time period is $\left[t_{1},\left(t_{2}-t_{1}\right) / 2\right]$, and the time period corresponding to the sweeping phase is $\left[\left(t_{2}-t_{1}\right) / 2, t_{2}\right]$.

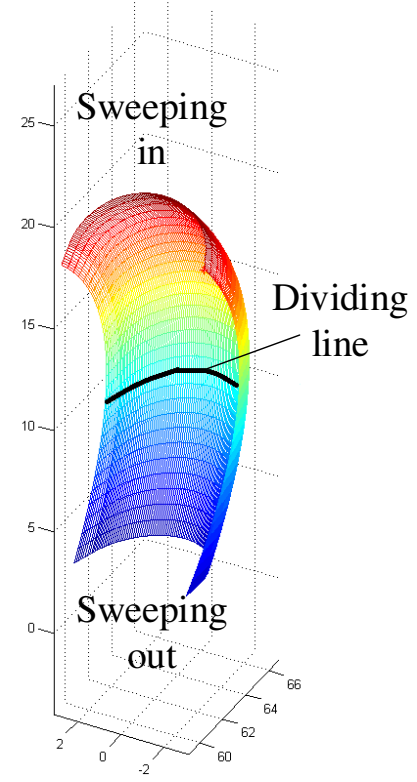

Figure 12 Schematic diagram of division of the edge-sweeping surface

As shown in Figure 13, the cutting process of the single gullet is divided into three states according to the different positions of the initial contact point of the edgesweeping surface and the tooth surface of the workpiece along the tooth width direction, that is, the plunging state, the full cutting state and the cutting out. state. The plunging state includes all cases from the upper end surface of the edge-sweeping surface to the upper end surface of the tooth groove to the edge of the edge-sweeping surface just touching the upper end surface of the tooth groove, as shown in Figs. 13(a) and (b); The sweeping boundary line is in all cases between the upper and lower end faces of the tooth groove, as shown in Figures 13(b) and (c); the cut-out state includes the edge of 
the edge-sweeping surface just touching the lower end of the tooth gap and all subsequent The situation is shown in Figures 13(c) and (d).

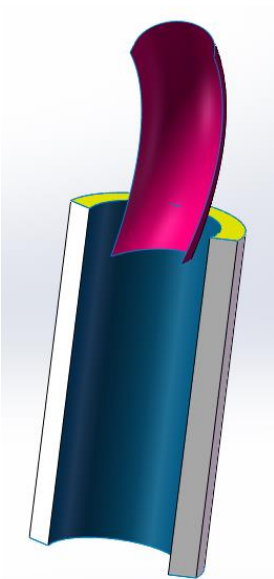

(a)

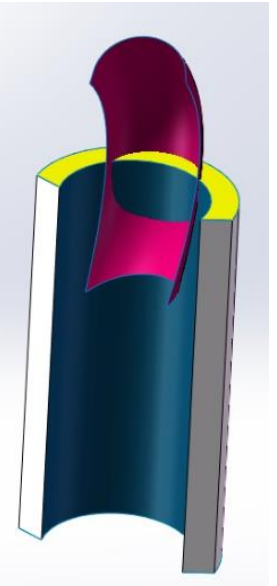

(b)

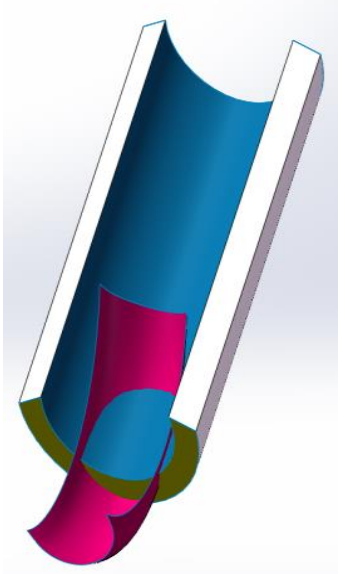

(c)

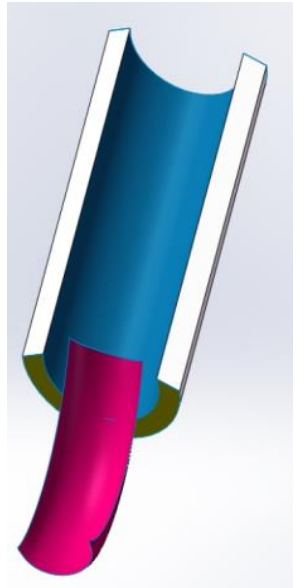

(d)

Figure13 Different cutting states during gear slicing process: (a) the edge-sweeping surface just touching the upper end of the tooth groove; (b) the dividing line of the edge-sweeping surface just touching the upper end of the tooth groove; (c) the dividing line of the edge-sweeping surface just touching the lower end of the tooth groove; (d) the edge-sweeping surface just off the lower end of the tooth groove;

During the gear slicing process, the undeformed chips are the closed space formed by the workpiece tooth groove end face, the workpiece tooth surface and the edgesweeping surface. The difference in the three cutting states results in different processes and specific shapes for the chips produced. For the plunging state, the undeformed chips are obtained by the upper end surface of the workpiece cogging, the tooth surface obtained by the previous cutting process, and the edge-sweeping surface. The shape of the chip mainly depends on the initial surface of the blade and the upper end of the workpiece. The moment of contact can be theoretically divided into three situations. In the first case, the sweeping phase of the edge-sweeping surface intersects the workpiece to obtain the chip. Since the relative rotational motion speed of the slice tool and the gear workpiece is much larger than the feed speed of the gear workpiece along the workpiece axis direction, the edge-sweeping surface is divided. The boundary line cannot touch the upper end surface of the workpiece cogging, so the first case does not exist in the machining of the caries; the second case is that the blade boundary or the sweeping phase intersects the workpiece to obtain the chip; the third case is the edgesweeping phase that intersects the workpiece to obtain the chip. In summary, there are only two cases in the cutting phase of the gear slicing process. 
For the full cutting state, the undeformed chips are obtained by the intersection of the $j$-th edge sweeping surface, the $j+1$ th edge-sweeping surface, and the tooth surface obtained by the previous cutting process. Irrespective of the influence of chip deformation, the shape of the undeformed chips obtained in the full cutting state is substantially the same, and the shape of the chips mainly depends on the relative position of the edge-sweeping surface family and the gear workpiece. For the cut-out state, the undeformed chips are obtained by intersecting the $i$-th edge-sweeping surface, the lower surface of the workpiece cogging, the tooth surface obtained by the previous cutting process, and the $i+1$ th edge sweeping surface. The shape of the chip mainly depends on the tooth width of the workpiece cogging, the tooth surface obtained by the previous cutting process, and the relative position of the edge-sweeping surface family and the gear workpiece.

It can be seen from the above analysis that in the cut-in state and the cut-out state, the upper and lower end faces of the workpiece tooth grooves serve as geometric boundaries of the undeformed chips, so that the geometry of the undeformed chips is incomplete and complicated. In addition, most of the cutting conditions during the gear slicing process are completely cut. Therefore, the main research on the undeformed chips in the full cutting state is studied here.

The arc gear is machined by the ATST-ERA as the carrier, and the tool, workpiece and machining parameters are shown in Table 1. Taking a tooth groove $E_{i}$ of the gear workpiece as an example, during the machining process of the tooth, the $p-1$ tooth cutting groove $E_{i-1}$, the $p$-th tooth cutting tooth groove $E_{i,}$, the $p+1$ th tooth cutting tooth groove $E_{i+1}$, and after the time $t$, the $q$-th tooth cutting the tooth groove $E_{i}$. With the $p$ th tooth contact slot $E_{i}$ as the initial time, the $q$-th tooth starts to contact the slot $E_{i}$ after the workpiece rotates one revolution, and the required time is $t$.

$$
t=\frac{60}{n_{1}}=\frac{2}{11} \mathrm{~s}
$$

According to the geometric relationship, the chip length along the tooth width direction is:

$$
L=v_{1} \times t=\frac{f n_{1} t}{60}=0.15 \mathrm{~mm}
$$

In the formula, $n_{1}$ represents the workpiece rotation speed, $v_{1}$ represents the workpiece feed speed, and $f$ represents the feed rate. 
Table 1 Correlative parameters of gear slicing by the ATST-ERA

\begin{tabular}{cccc}
\hline Parameter & Value & Parameter & Value \\
\hline Tooth number of cutter & 22 & Arc radius of workpiece & $5.8 \mathrm{~mm}$ \\
Helix angle of cutter & $20^{\circ}$ & Distribution circle diameter of workpiece & $128 \mathrm{~mm}$ \\
Rake angle of cutter & $10^{\circ}$ & Cutting depth & $0.3 \mathrm{~mm}$ \\
Clearance angle of cutter & $6.3^{\circ}$ & Feed per revolution & $0.15 \mathrm{~mm} / \mathrm{r}$ \\
Crossed-axes angle & $20^{\circ}$ & Rotate speed of cutter & $700 \mathrm{r} / \mathrm{min}$ \\
Tooth number of workpiece & 40 & Rotate speed of workpiece & $385 \mathrm{r} / \mathrm{min}$ \\
\hline
\end{tabular}

The chip thickness $s(t, h)$ is the thickness of the undeformed chips in the workpiece coordinate system at different width positions, and $s(t, h)$ is composed of $s_{1}(t, h)$ and $s_{2}(t, h)$, ie

$$
s(\mathrm{t}, \mathrm{h})=\left\{\begin{array}{c}
s_{1}(\mathrm{t}, \mathrm{h})=G(\mathrm{x}, \mathrm{y}, \mathrm{z})-\boldsymbol{r}_{j+1}(r, \varphi), \mathrm{t}_{1} \leq t \leq t_{2} \\
s_{2}(\mathrm{t}, \mathrm{h})=\boldsymbol{r}_{j}(r, \varphi)-\boldsymbol{r}_{j+1}(r, \varphi), \mathrm{t}_{2} \leq \mathrm{t} \leq \mathrm{t}_{3}
\end{array}\right.
$$

In the formula, $t_{1}$ indicates the moment when the $j+1$ th blade sweep surface is just in contact with the tooth surface obtained by the previous cutting process, and $t_{2}$ indicates the moment when the $j$-th blade sweep surface is just in contact with the tooth face obtained by the previous cutting process. , $t_{3}$ represents the moment when the $j$-th edge sweeping surface is in contact with the finally formed tooth surface.

According to the basic theory of differential geometry, the chip volume corresponding to the spatial path of the cutting edge can be obtained:

$$
V=\iint_{D} \mathrm{~s}(t, h) \cdot v_{1} d t d h=\int_{0}^{t_{4}} \int_{0}^{h(t)} s(t, h) \cdot v_{1} d h d t
$$

In this formula, $D=\left\{(t, \mathrm{~h}) \mid 0 \leq t \leq t_{4}, 0<h \leq h(t)\right\}, \mathrm{t}_{4}=\frac{2}{11} \mathrm{~s}, h(t)$ represents the width of the undeformed chip.

According to the spatial geometric relationship, the undeformed chips in the fully cut state are the spatial geometry obtained by the intersection of the tooth surface obtained by the previous cutting process, the $j$-th edge sweeping surface and the $j+1$ th edge-sweeping surface, that is

$$
\left\{\begin{array}{c}
\boldsymbol{r}_{j}(r, \varphi)=\boldsymbol{T} \cdot \boldsymbol{r}_{j}(r) \\
\boldsymbol{r}_{j+1}(r, \varphi)=\boldsymbol{T} \cdot \boldsymbol{r}_{j+1}(r) \\
G(x, y, z)=0
\end{array}\right.
$$


According to the above formula, the shape of the undeformed chips can be obtained by means of the three-dimensional modeling software, as shown in Figure14.

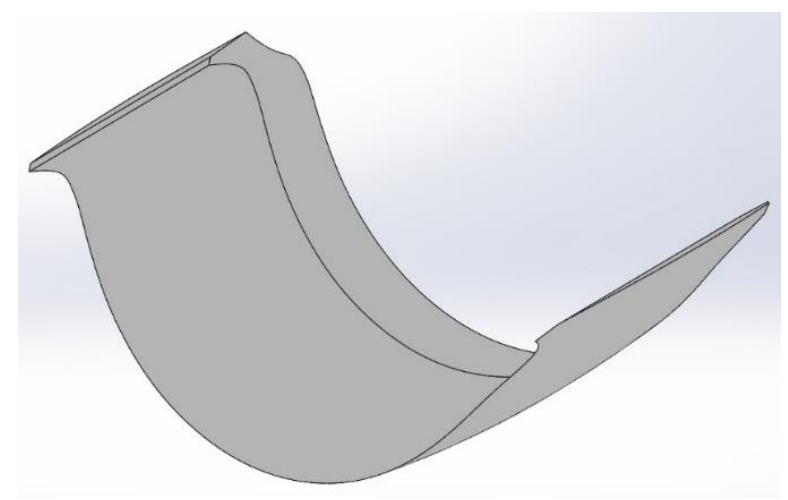

Figure 14 Undeformed chip

\subsection{Experimental verification}

Using the geometry model of undeformed chip, the undeformed chips with different cutting parameters can be obtained. For the different cutting parameters in Table 2, the undeformed chips got by simulation calculation are shown in Figure 15. In order to verify the geometric model of the undeformed chips, the cutting parameters in Table 2 were selected, and a trial cutting experiment was carried out on the cutting machine tool to collect chips. As mentioned above, for the complete cutting state, the shape of the chips obtained by cutting the workpiece material with the cutting tool teeth is basically the same. Therefore, the chips with common shapes are collected in the experiment as a typical example. The chips produced by different cutting parameters are shown in Figure 16.

Table 2 Different slicing parameters of gear slicing by the ATST-ERA

\begin{tabular}{ccccc}
\hline $\begin{array}{c}\text { Serial } \\
\text { number }\end{array}$ & $\begin{array}{c}\text { Cutting depth } \\
/(\mathrm{mm})\end{array}$ & $\begin{array}{c}\text { Rotate speed of } \\
\text { cutter } /(\mathrm{r} / \mathrm{min})\end{array}$ & $\begin{array}{c}\text { Feed per revolution } \\
/(\mathrm{mm} / \mathrm{r})\end{array}$ & $\begin{array}{c}\text { Rotate speed of } \\
\text { workpiece } /(\mathrm{r} / \mathrm{min})\end{array}$ \\
\hline 1 & 0.1 & 450 & 0.1 & 247.5 \\
2 & 0.2 & 600 & 0.2 & 330 \\
3 & 0.3 & 700 & 0.15 & 385 \\
\hline
\end{tabular}



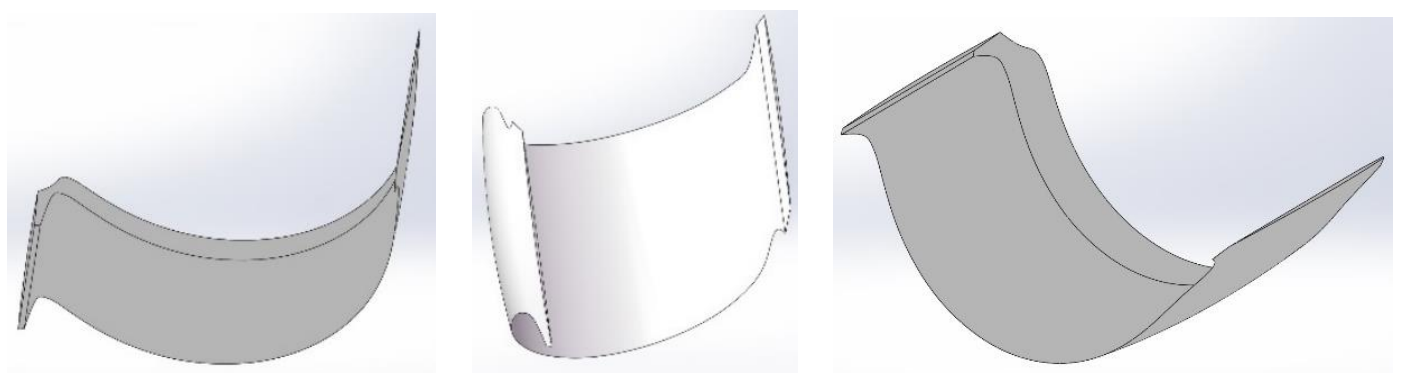

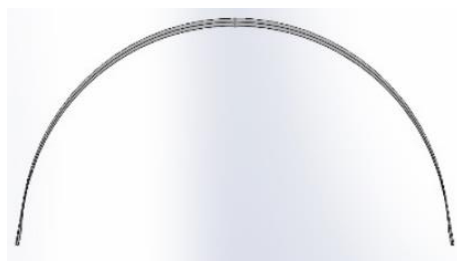

(a)

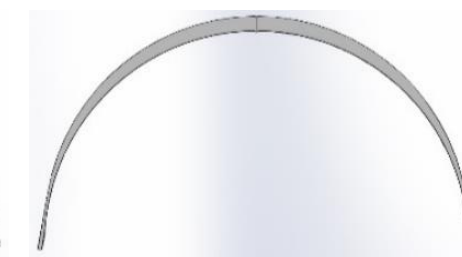

(b)

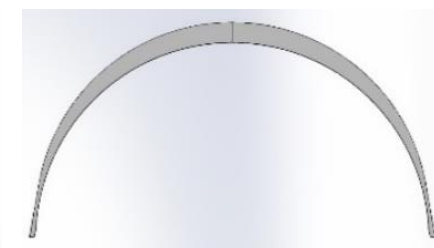

(c)

Figure 15 Undeformed chip obtained by simulation calculation

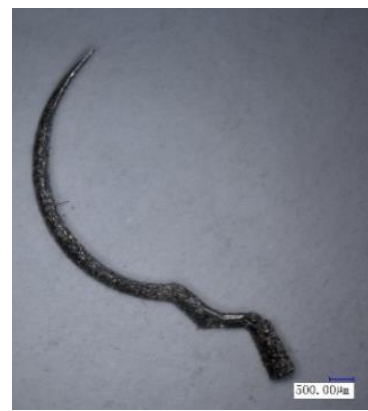

(a)

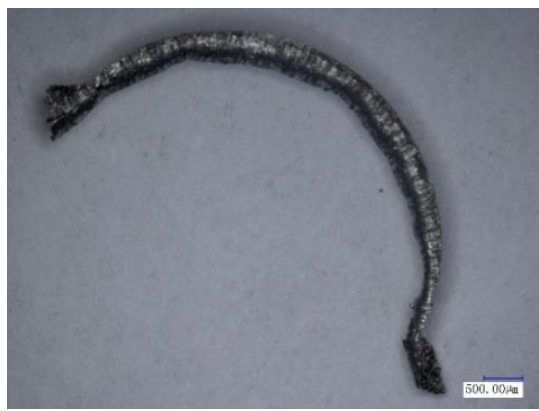

(b)

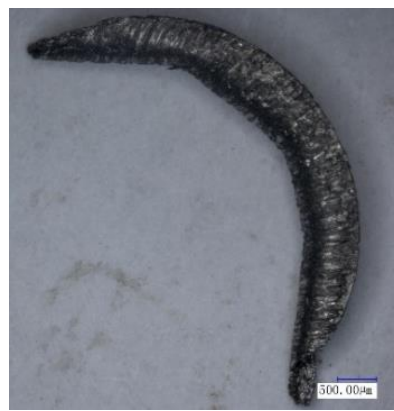

(c)

Figure 16 The experimentally collected deformed chips

As can be seen from Figure 15, the contour of the undeformed chip is a special free curve, and its projection on the plane is approximately crescent-shaped. As for the chip thickness, when the cutting parameters are unchanged, the chip thickness in the cutting-in and cutting-out stages is the smallest, and the chip thickness is the largest in the part corresponding to the boundary line of the blade-sweeping surface. In addition, when the cutting parameters change, the chip thickness increases as the depth of cut increases. Figure 16 shows that the chips collected in the experiment are all deformed chips, and the main deformation form of the chips is curling. The chips after curling are also approximately crescent-shaped. As the cutting depth increases, the chip thickness also shows an increasing trend. Through the above qualitative comparative analysis, it can be seen that the shape of the chip obtained by the simulation calculation is basically the same as the shape of the chip collected by the experiment. The chip shape obtained 
by the experiment is partially bent or broken which is caused by the extrusion or the friction during the chip-forming process. In addition, the thickness changes of the chips got by the simulation calculation and the experiment are basically the same, and both have a positive correlation with the depth of cut.

In order to quantitatively analyze and compare the simulated and experimental chips, the measurement function in Solidworks and the optical microscope of Keyence VX-1000 were used to measure the chip contour radius, arc length and chip thickness obtained by simulation calculation and experimental collection. In Solidworks, the contour radius measurement value is obtained by measuring the undeformed chip contour surface, and the arc length and chord length are obtained by measuring the contour curve; the contour radius and chord length of the chip are directly obtained by measuring the curled chip contour with the help of an optical microscope. The arc angle corresponding to the chip profile curve is got by measuring the chip profile after curling deformation, and then the arc length value of the chip is calculated, as shown in Figure 17.
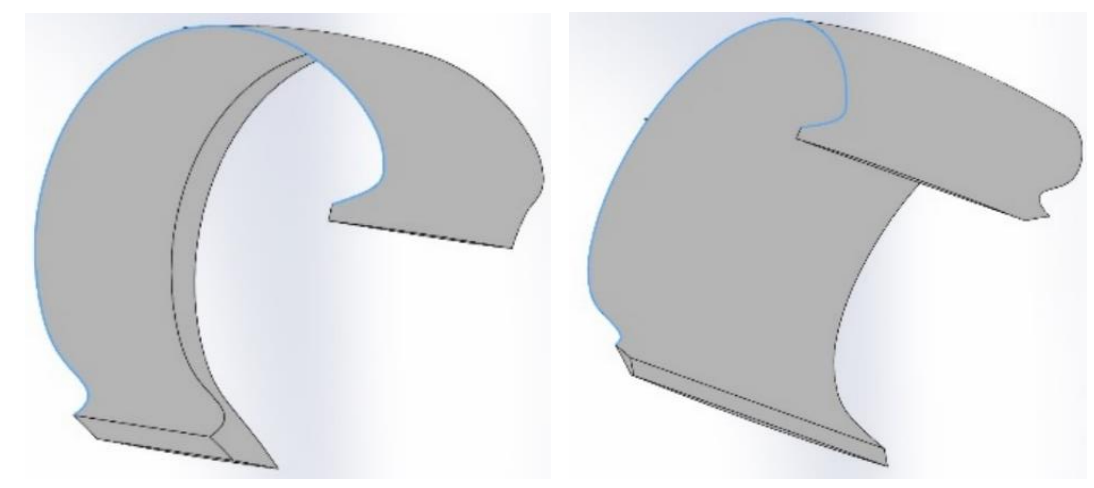

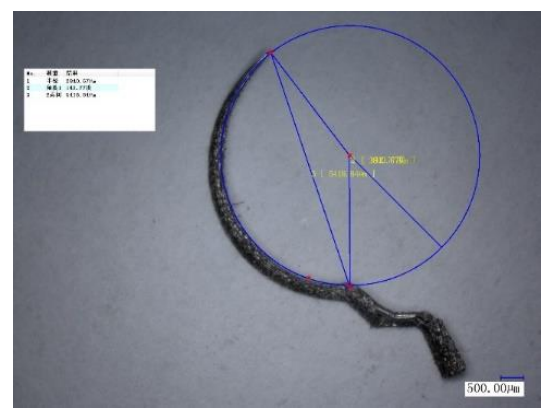

(a)

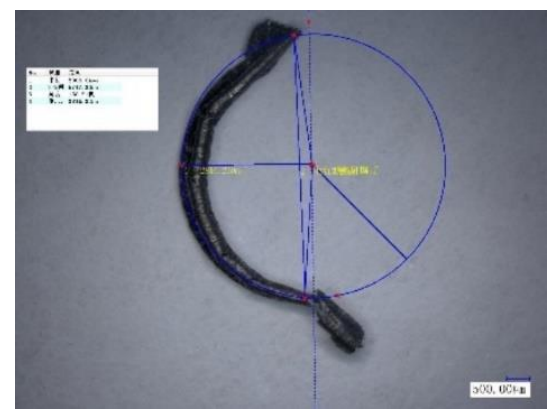

(b)

Figure 17 Measurement results of chip shape parameters under different slicing parameters

For the undeformed chips obtained by the simulation calculation, the undeformed chips are sectioned in Solidworks, and then the maximum chip thickness is measured; 
for the deformed chips obtained by the experiment, the curled chips are first spread out, and then with the help of the optical microscope, the thickness value of the deformed chip is obtained by measuring the distance between the upper and lower surfaces of the chip, as shown in Figure 18. For three groups of different cutting parameters, the measured simulation and experimental chip shape parameter values (including: contour radius, arc length, chord length and chip thickness) are shown in Table 3.
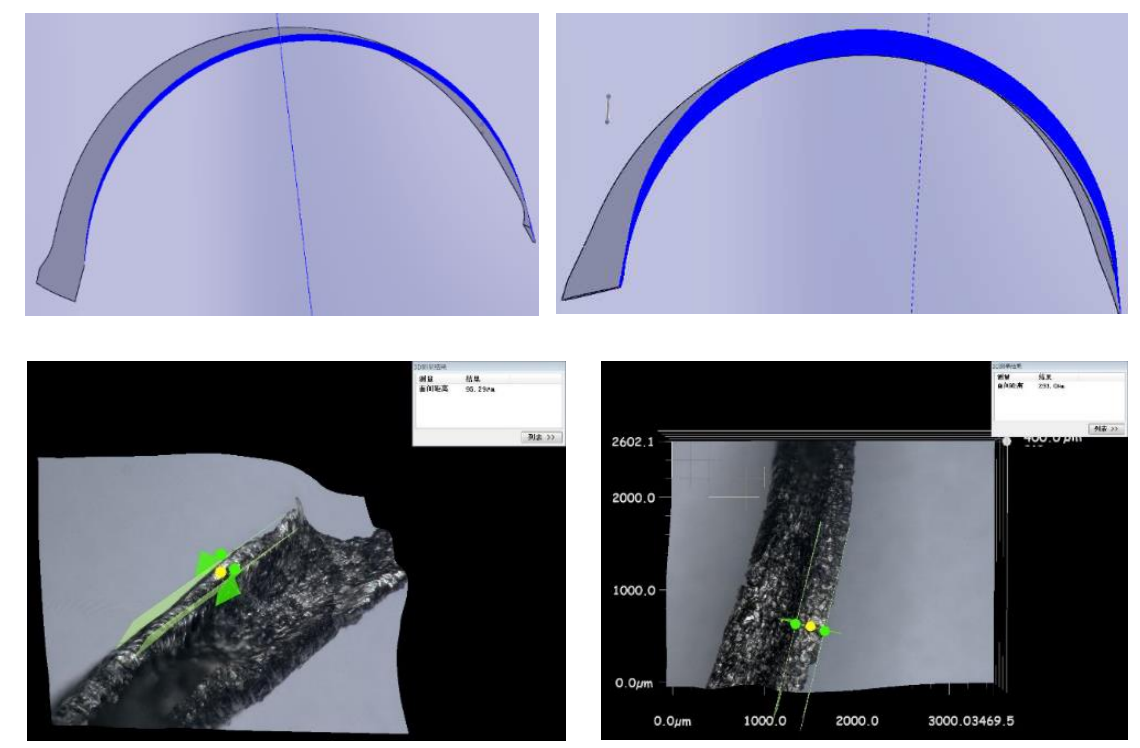

(a)

(b)

Figure 18 Measurement results of chip thickness under different slicing parameters

Table 3 Measurement results of chip shape parameters obtained by simulation and experiment under different slicing parameters

\begin{tabular}{ccccccccc}
\hline \multirow{2}{*}{$\begin{array}{c}\text { Serial } \\
\text { number }\end{array}$} & \multicolumn{2}{c}{ Outline radius $(\mathrm{mm})$} & \multicolumn{2}{c}{ Arc length $(\mathrm{mm})$} & \multicolumn{2}{c}{ Chord length $(\mathrm{mm})$} & \multicolumn{2}{c}{ Chip thickness (mm) } \\
& Simulation & Experiment & Simulation & Experiment & Simulation & Experiment & Simulation & Experiment \\
\hline 1 & 2.900 & 2.8407 & 10.0494 & 7.0785 & 6.3160 & 5.4188 & 0.0907 & 0.0953 \\
2 & 2.900 & 2.8696 & 9.6259 & 8.4396 & 6.2137 & 5.7172 & 0.1901 & 0.1945 \\
3 & 2.900 & 2.9494 & 9.9241 & 7.4574 & 6.3702 & 5.6076 & 0.2902 & 0.2930 \\
\hline
\end{tabular}

Comparing and analyzing the measurement results of the above-mentioned chip contour radius, it can be found that the contour radius of the chip obtained by simulation calculation and experiment under different cutting parameters are very close, and the maximum deviation is $2.04 \%$. The contour radius of the simulated chip does not change with the change of the cutting parameters, and both are $2.9 \mathrm{~mm}$, indicating that the 
contour radius of the chip has no relationship with the cutting parameters, but is equal to the tooth radius of the cutting tool. The contour radius of the experimental chip fluctuates around $2.9 \mathrm{~mm}$. The reason why the $2.9 \mathrm{~mm}$ don't become constant is due to chip deformation and measurement errors.

A comparative analysis of the above-mentioned chip arc length and chord length measurement results shows that the contour arc length and chord length of the chip are related to the feed amount and the relative speed between the tool and the workpiece. Other cutting parameters remain unchanged. The greater the speed, the greater the arc length and chord length of the chip contour curve, and the greater the feed, the smaller the arc length and chord length value of the chip contour curve. The arc length and chord length of the chips obtained by simulation and experiment under different cutting parameters are quite different. The main reason is that the chips obtained in the experiment are curled, squeezed or stretched and deformed, which causes the chips to tear away from somewhere and are difficult to maintain complete chip shape.

Comparing the measurement results of the above-mentioned chip thickness, it can be found that the thickness of the chips obtained by the simulation calculation and the experiment under different cutting parameters is basically the same, and the maximum deviation is $5.07 \%$. The main reason is that the chips obtained in the experiment are squeezed and deformed, which leads to the increase of the chip thickness. In addition, the chip thickness is mainly related to the depth of cut and increases with the increase of the depth of cut.

Based on the above comparative analysis, it can be seen that ignoring the influence of chip deformation, the shape of the chip obtained by simulation calculation and experiment is basically the same, which proves that the geometric model of undeformed chip is correct. In addition, the chip contour radius depends on the tooth radius of the cutting tool. The chip thickness is positively related to the cutting depth. The arc length and chord length of the chip contour curve are positively related to the relative speed between the tool and the workpiece, and is negatively related to the feed-up energy.

\section{Analysis of Rake Face Wear Based on Cutting Edge Load}

According to the particularity and complexity of the slicing technology, the load moments of the caries are changed during the process of caries machining and the load distribution is extremely uneven. In addition, since the slicing is performed under dry cutting conditions, the cutters are more susceptible to wear. Therefore, this section studies the load distribution and wear analysis of the cutting edge of the tooth cutter. 
According to the empirical formula of cutting load proposed by scholar Kienzle ${ }^{[32]}$, the cutting thickness and the area of the cutting layer directly affect the cutting load during the cutting process. During the cutting process, the force of the cutting edge against the chip and the force of the chip against the cutting edge are equal and opposite, so the calculation of the load of the cutting edge can be converted into the calculation of the chip load. The chip load can be calculated by the following formula.

$$
F=A \cdot \frac{k_{c}}{h^{u}}
$$

Where $k_{\mathrm{c}}$ represents the cutting force per unit area when the nominal section thickness and the nominal width of the chip are $1 \mathrm{~mm}, A$ represents the area of the cutting layer, $h$ represents the cutting thickness, and $u$ is a constant value coefficient, indicating the influence of the cutting thickness $h$ on the cutting load.

According to the chip forming principle of the gear slicing, the chip formed by the edge-sweeping surface sweeping the workpiece tooth groove reflects the shape of the cutting layer of the cutting edge of the slice tool at different times, as shown in Figure 19. According to the formula 16 , the chip thickness $s(t, h)$ of the cutting edge of the slice tool can be extracted at any time, as shown in Figure 20. The contour of the cutting edge of the cutting edge of the slice tool is mainly crescent-shaped. According to the shape of the cutting layer, the area of the cutting layer micro-element at any point on the cutting edge determined by the time $t$ can be calculated as

$$
\mathrm{d} A=\left|\frac{\mathrm{d} E_{h}(j, t)}{\mathrm{d} t}\right| s(t, h) \mathrm{d} t
$$

The area of the micro-element at a certain point of the cutting edge is shown in Figure 21.

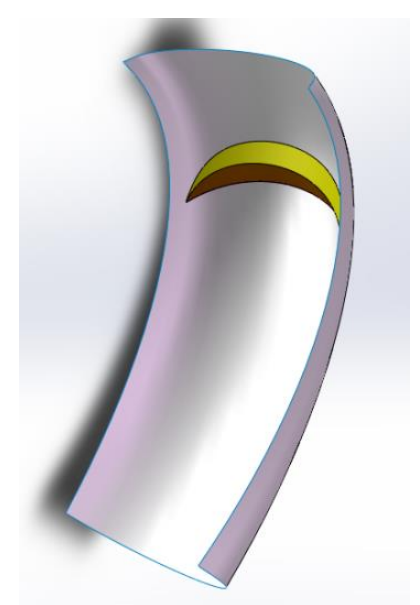


Figure 19 Space sweeping trail of edge-sweeping surface

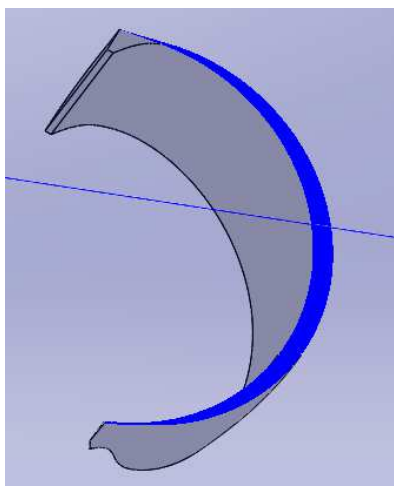

(a)

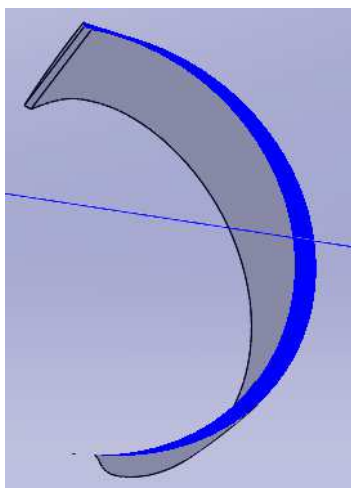

(b)

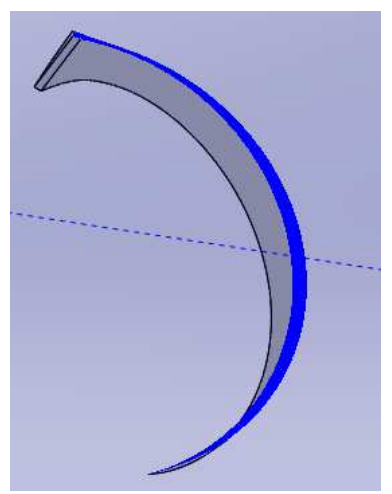

(c)

Figure 20 Chip thickness corresponding to the cutting edge at different times

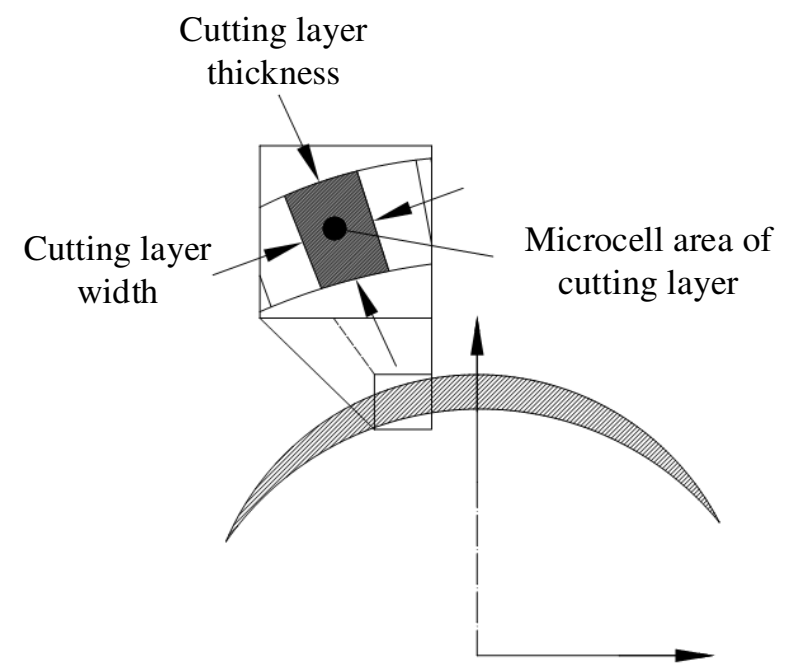

Figure 21 Microcell area at a certain point of the cutting edge

According to the calculation formula of the Kienzle cutting load, the micro-area cutting load at any point on the cutting edge can be derived:

$$
F^{i, j}(\mathrm{t}, \mathrm{h})=\left(\left|\frac{d E_{h}{ }^{\prime}(j, t)}{d t}\right| s(t, h) d t\right)\left(\frac{k_{c}}{s(t, h)^{u}}\right)
$$

Then, the cutting load per unit length of the cutting edge is:

$$
L^{i, j}(\mathrm{t}, \mathrm{h})=(s(t, h))^{1-u} k_{c}
$$

In the formula, $L^{i, j}(t)$ is the cutting load at different positions determined by the cutting edge of the $j$-th tooth of the arc tooth cutter on the $i$-th groove is determined with time $t$. By integrating the pair of $t$ and taking a linear average over the cutting time 
range $t_{\text {start }} \sim t_{\text {end }}$, the cutting load of the cutting edge of the arc tooth cutter during the cutting of a certain chip can be obtained. The cutting load in the cutting time range $t_{\text {start }} \sim t_{\text {end }}$ is:

$$
\sigma^{i, j}(t)=\frac{\int_{t_{\text {stat }}}^{t_{\text {end }}} L^{i, j}(t, h) d t}{t_{\text {end }}-t_{\text {start }}}
$$

The linear value of the cutting edge load of the different teeth of the arc tooth cutter can more accurately reflect the comprehensive loading condition of the different cutting edges of the arc tooth cutter. The integrated load of the blade is:

$$
\sigma^{i}(t)=\frac{1}{j} \sum_{j} \sigma^{i, j}(t)=\frac{1}{j} \sum_{j} \int L^{i, j}(t, h) d t
$$

Select the parameters in Table 1 to calculate the cutting edge load of the ATSTERA. The calculated coefficients $K_{c}$ and $u$ in the Kienzle formula are related to the workpiece material and are usually obtained experimentally. The workpiece material selected here is QT450-10, the parameter value in reference ${ }^{[33]}$, taking $K_{c}=1451 \mathrm{~N} / \mathrm{mm} 2$, $u=0.17$. During the machining process of the caries, there are differences in the cutting process of the different teeth of the arc tooth cleaver. In order to get closer to the real situation, the teeth of No. 3, No. 6 and No. 15 of the arc knives are randomly selected for calculation. The calculated load spectrum of the three teeth is shown in Figure 22. It can be seen that the load distribution has a tendency to gradually decrease toward the both sides in the middle, and the position where the load is the largest is at the left-toleft position in the middle.

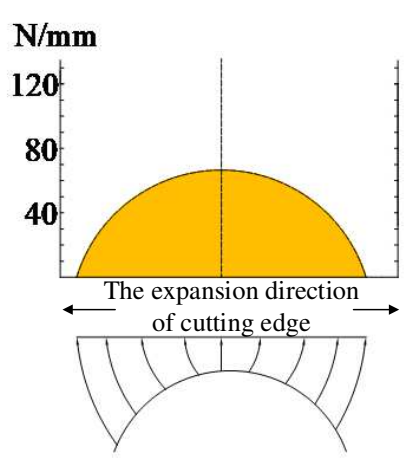

(a) No. 3 cutter teeth

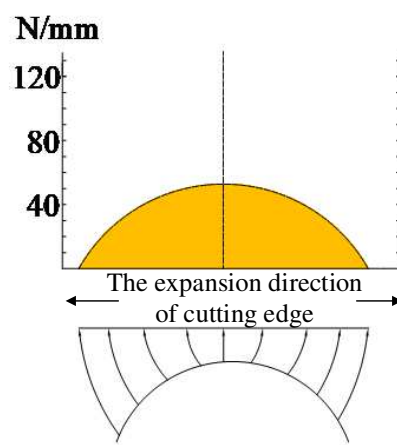

(b) No. 6 cutter teeth

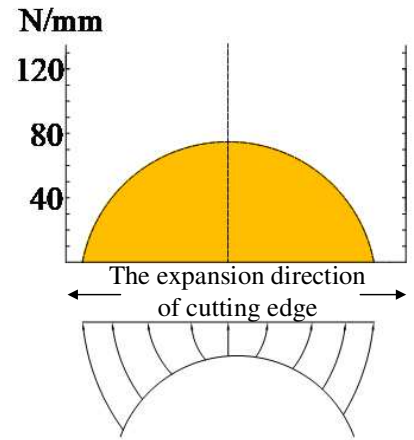

(c) No. 15 cutter teeth

Figure 22 Load spectrum of No. 3, No. 6 and No. 15 cutter teeth 
Considering that the cutting load is the main cause of tool wear, the parameters in Table 2 are selected for the cutting experiment. After testing 100 gear workpieces, the optical microscope of KEYENCE VX-1000 is used to photograph the rake faces of arc cutters No. 3, No. 6 and No. 15, and the wear conditions are shown in Figure 23. It can be seen from the figure that the left part of the cutting edge wears the most severely and gradually weakens to the two sides, and the wear on the left part is more serious than the wear on the right part. Although the number of gear workpieces cut by the cutting tool is small and the wear of the rake face of the cutting teeth is not too serious, there is still crater wear near the cutting edge on the No. 15 cutting tooth, as shown in Figure 24.
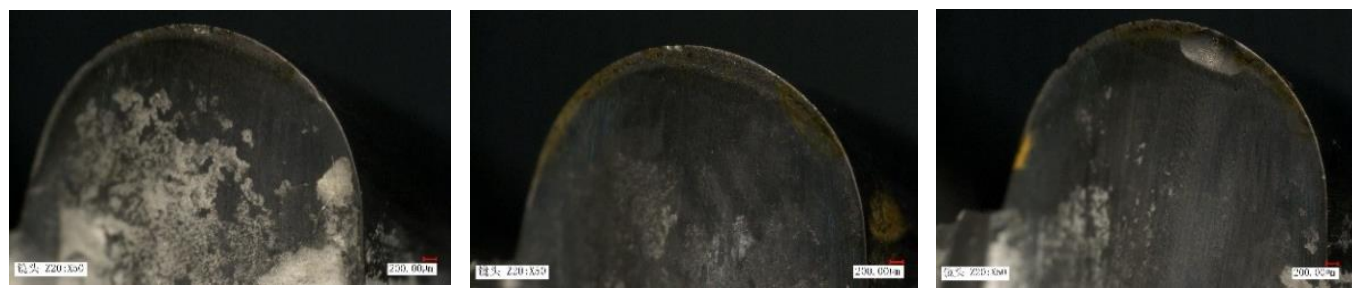

Figure 23 Schematic diagram of wear on rake face of No. 3, No. 6 and No. 15 cutter teeth
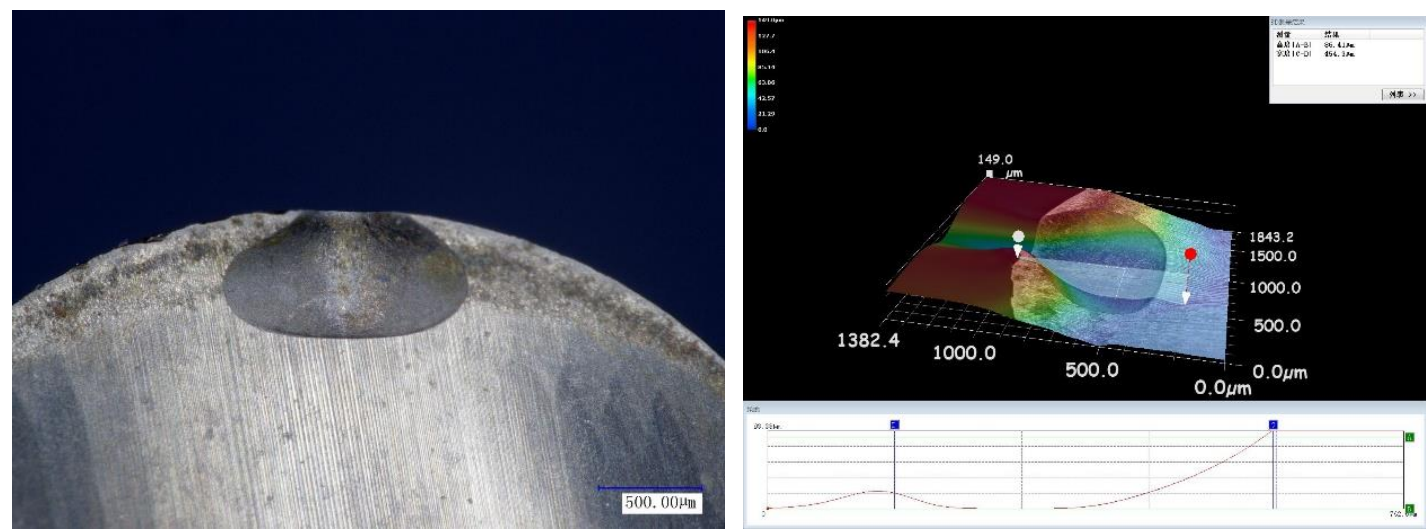

Figure 24 Schematic diagram of crater wear on rake face of No. 15 cutter tooth

In order to further analyze the relationship between the cutting edge load distribution and the tool wear, the load spectrum of the No. 15 cutter cutting edge is compared with the rake face wear, as shown in Figure 25. It can be seen from the figure that the wear of the cutting edge in the middle left position is the most serious, and the degree of wear to the left side of the cutting edge is gradually lower, which is also consistent with the average load distribution of the cutting edge. Therefore, the wear amount of the arc tooth cutter is positively correlated with the load distribution of the cutting edge. 


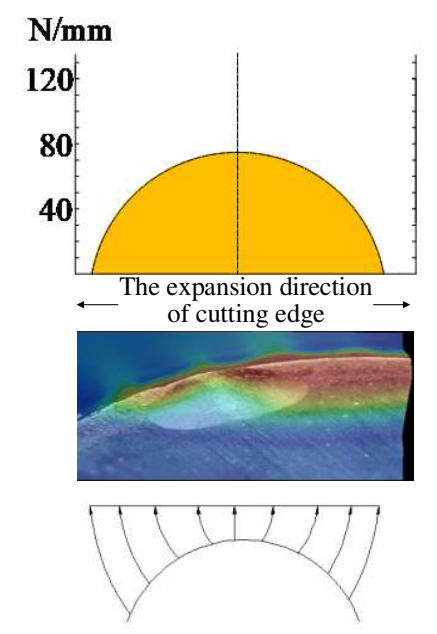

Figure 25 Load spectrum and wear of No. 15 cutter tooth

\section{Conclusion}

Aiming at the particularity of the slicing process with ATST-ERA, this paper proposes a chip forming modeling method. By analyzing the common characteristics of the cutting process, a unified mathematical abstract expression of the cutting process is established and cutting edge, edge-sweeping surface, edge-sweeping surface family and tooth surface in the slicing process, the parametric model of slicing process of the ATST-ERA is developed. Then establish the undeformed chip geometry model for the cutting process, and use this model to obtain the undeformed chip shape with different cutting parameters. Through a comprehensive comparative analysis with the chips obtained from the cutting experiment, it is proved that the geometry model of the chips is correct. In addition, the chip contour radius depends on the tooth radius of the cutting tool. The chip thickness is positively related to the cutting depth. The arc length and chord length of the chip contour curve are positively related to the relative speed between the tool and the workpiece, and the follow-up feed is negatively related. Based on the chip geometry model, the KV cutting force empirical formula is used to calculate the cutting edge load of the equal rake angle circular arc cutter. Through a comparative analysis with the rake face wear, it is determined that the cutting edge load and the rake face wear have positive relationship. The research results reveal the chip forming mechanism of the ATST-ERA, which provides a theoretical basis for chip shape control and tool wear compensation during cutting.

\section{Authors' contributions:}

Xiaoqiang Wu: Writing- Original draft preparation. 
Yongquan Jin: Data curation, Writing- Reviewing and Editing.

\section{Funding}

This work was supported by Natural Science Foundation of China (No. 51775374), Program for Young Talents of Science and Technology in Universities of Inner Mongolia Autonomous Region (No. NJYT-19-B15).

\section{Data availability}

The authors confirm that the data supporting the findings of this study are available within the article.

\section{Compliance with ethical standards}

Competing interests: The authors declare that they have no competing interests.

Ethical approval: Not applicable.

Consent to participate: Not applicable.

Consent to publish: Not applicable.

\section{References}

[1] Spath D, Huhsam A. Skiving for High-performance Machining of Periodic Structures [J]. Annals of the CIRP, 2002, 51(1): 472-475.

[2] Hartmut M, Olaf V. Robust Method for Skiving and Corresponding Apparatus Comprising a Skiving Tool [P]: USA, 20120328384A1, 2012.

[3] Hartmut M, Olaf V. Semi-completing Skiving Method and Device Having Corresponding Skiving Tool for Executing a Semi-completing Skiving Method [P]: USA, 20130071197A1, 2013.

[4] Stadtfeld H, Donnan J, Robert T. Skiving of Cylindrical Gears [P]: USA, WO2014US34813, 2014.

[5] Stadtfeld H, Donnan J, Robert T. Skiving of Cylindrical Gears [P]: USA, WO2014US34813, 2014.

[6] Li J, Chen X C, Zhang H Y. Slicing Technology for Cylindrical Gears [J]. Journal of Mechanical Engineering, 2011, 47(19): 193-198.

[7] Guo E K, Hong R J, Huang X D, et al. A Novel Power Skiving Method Using the Common Shaper Cutter [J]. International Journal of Advanced Manufacturing Technology, 2016, 83(1): 157165.

[8] Zheng G, Mao S M, Li X E, et al. Research on the Theoretical Tooth Profile Errors of Gears Machined by Skiving [J]. Mechanism and Machine Theory, 2016, 97: 1-11.

[9] Chen X C, Li J, Lou B C. A Study on the Design of Error-free Spur Slice Cutter [J]. International 
Journal of Advanced Manufacturing Technology, 2013, 68(1-4): 727-738.

[10] Li J, Wang P, Chen X C, et al. A Study on the Optimal Selection of Spur Slice Cutter Parameters and Machining Parameters [J]. International Journal of Advanced Manufacturing Technology, 2016, 82(1): 407-417.

[11] Li J, Lou B C, Chen X C. Structural Design of Slice Cutter Based on Free-form Surface [J]. Journal of Mechanical Engineering, 2014, 50(17): 157-164.

[12] Wang P, Li J, Jin Y Q. A Study on the Design of Slicing Cutter for Cycloid Gear based on Conjugate Theory [J]. International Journal of Advanced Manufacturing Technology, 2018, 98(5-8): 2057-2068.

[13] Volker S, Chirsttoph K, Hermann A. 3D-FEM Modeling of Gear Skiving to Investigate Kinematics and Chip Formation Mechanisms [J]. Advanced Materials Research, 2011, 223: 46-55. [14] Mazak J, Klocke F, Bergs T, et al. Simulation-based process analysis for discontinuous cutting of generated bevel gears [J]. Proc IMechE Part C: Journal of Mechanical Engineering Science, 2019, doi: $10.1177 / 0954406219845409$.

[15] Tapoglou. Calculation of non-deformed chip and gear geometry in power skiving using a CADbased simulation [J]. International Journal of Advanced Manufacturing Technology, 2018, doi: https://doi.org/10.1007/s00170-018-2790-3.

[16] McCloskey P, Katz A, Berglind L, et al. Chip geometry and cutting forces in gear power skiving [J]. CIRP Annals - Manufacturing Technology, 2019, https://doi.org/10.1016/j.cirp.2019.04.085.

[17] Uysal A, Jawahir I S. A slip-line model for serrated chip formation in machining of stainless steel and validation [J]. International Journal of Advanced Manufacturing Technology, 2018, doi: https://doi.org/10.1007/s00170-018-3136-x.

[18] Su R, Huang C, Xu L H, et al. Research on the serrated chip in the milling of compacted graphite iron by cemented carbide tool [J]. International Journal of Advanced Manufacturing Technology, 2018, 99(5-8): 1687-1698.

[19] Zang J, Zhao J, Li A H, et al. Serrated chip formation mechanism analysis for machining of titanium alloy Ti-6Al-4V based on thermal property [J]. International Journal of Advanced Manufacturing Technology, 2018, 98(1-4): 119-127.

[20] Ahmed Y S, Paiva J M, Veldhuis S C. Characterization and prediction of chip formation dynamics in machining austenitic stainless steel through supply of a high-pressure coolant $[\mathrm{J}]$. International Journal of Advanced Manufacturing Technology, 2019, doi: https://doi.org/10.1007/s00170-018-03277-7.

[21] Rakesh M, Datta S. Effects of Cutting Speed on Chip Characteristics and Tool Wear Mechanisms During Dry Machining of Inconel 718 Using Uncoated WC Tool [J]. Arabian Journal 
for Science and Engineering, 2019，44(9): 7423-7440.

[22] Molnar T G, Berezvai S, Kiss A M, et al. Experimental Investigation of Dynamic Chip Formation in Orthogonal Cutting [J]. International Journal of Machine Tools and Manufacture, 2019, 145: 103429 .

[23] Dou W, Cui G W, Yuan S W, et al. Analysis of Uncut Milling Chip Thickness Based on Trochoidal Tooth Path [J]. Journal of Mechanical Engineering, 2019, 55(7): 234-242.

[24] Nikawa M, Okada M, Mori H, et al. Evaluation of machinability in milling by controlling chip thickness using NC simulation [J]. Journal of Mechanical Science and Technology, 2018, 32 (10): $4851-4858$.

[25] Harry O O, Patxi A O, Pedro J A A. Analytical modeling of the uncut chip geometry to predict cutting forces in orthogonal centric turn-milling operations [J]. International Journal of Machine Tools and Manufacture, 2019, 144: 103428.

[26] Berenji, K R, Karagüzel U, Özlü E, et al. Effects of turn-milling conditions on chip formation and surface finish [J]. CIRP Annals - Manufacturing Technology, 2019, 68(1): 113-116.

[27] Bergmann B, Denkena B, Grove T, et al. Chip Formation of Rounded Cutting Edges [J]. International Journal of Precision Engineering and Manufacturing, 2019, 20(1): 37-44.

[28] Chen Y P, Cao H J, Li X G, et al. The Model of Spatial Forming with Multi-cutting-edge for Cylindrical Gear Hobbing and Its Application [J]. Journal of Mechanical Engineering, 2016, 52(9): 176-183.

[29] Chen Y P, Cao H J, Yang X. Research on Load Distribution Characteristic on the Cutting Edge in High Speed Gear Hobbing Process [J]. Journal of Mechanical Engineering, 2017, 53(15): 181187.

[30] Li B J, Cao H J, Yang X, et al. Contribution of chip deformations and crushes to tool failures in high-speed dry gear hobbing [J]. China Mechanical Engineering, 2019, 30(1): 71-78.

[31] Zhang R C. Research on Some Key Technologies of Hobbing Force Prediction of Cylindrical Gear [D]. Shenyang: Northeastern University, Phd thesis, 2016.

[32] Mustafa G, Ersan A, Ihsan K, et al. Investigation of the effect of rake angle on main cutting force [J]. International Journal of Machine Tools \& Manufacture, 2004, 44(9): 953-959.

[33] Chen Y P. Spatial Forming Model of High-speed Dry Hobbing by Interrupted Cutting with Multiple-cutting-edge and Its Application [D]. Chongqing: Chongqing University, Phd thesis, 2015. 
Figures

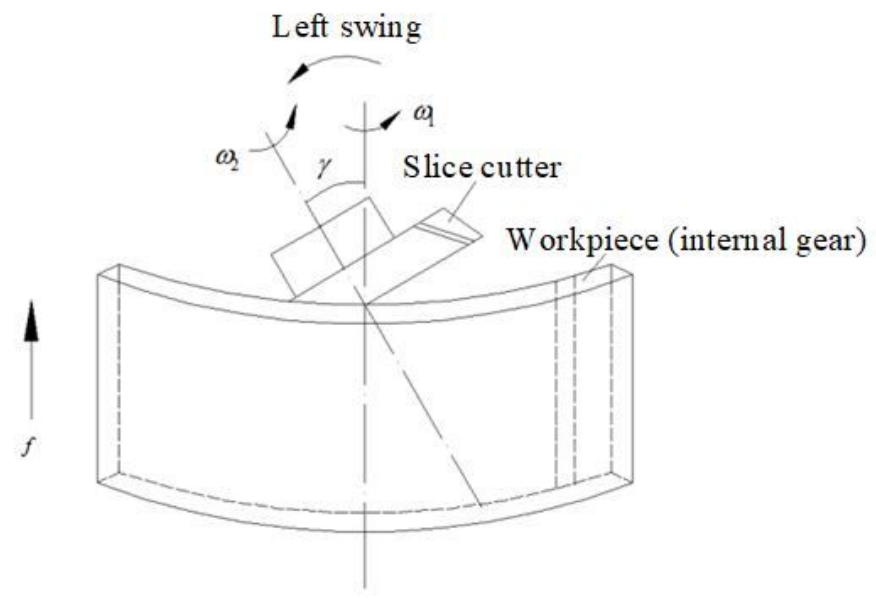

(a)

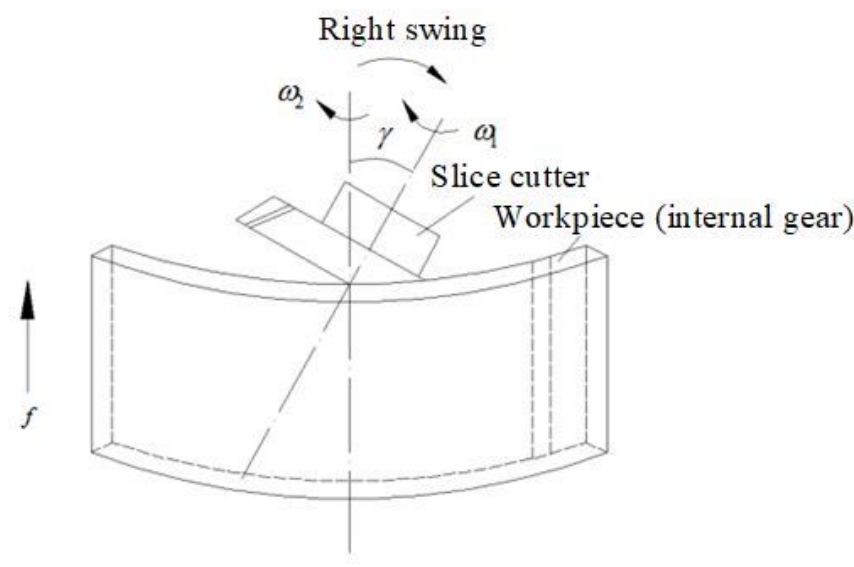

(b)

\section{Figure 1}

Slicing of the spur and internal gear

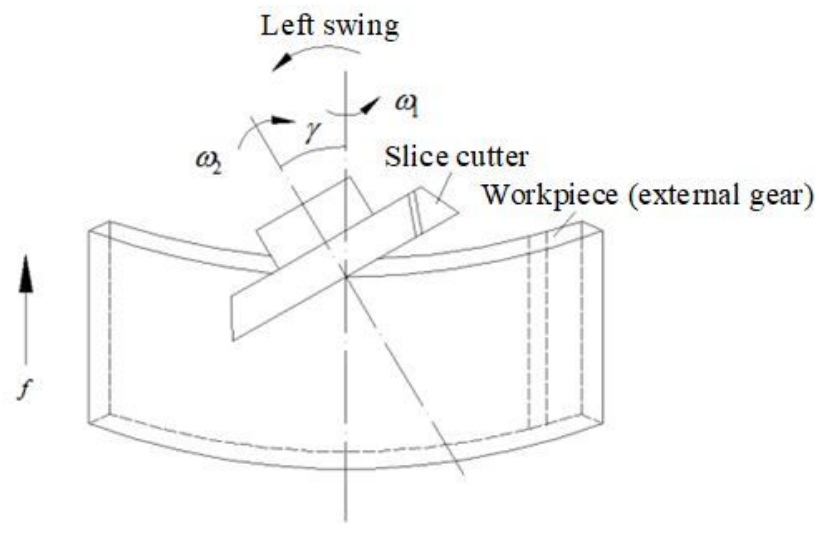

(a)

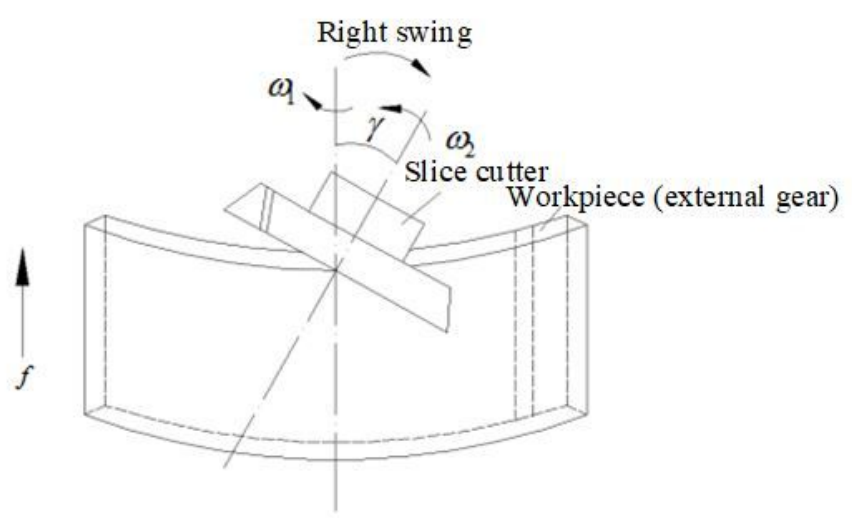

(b)

Figure 2

Slicing of the spur and external gear 


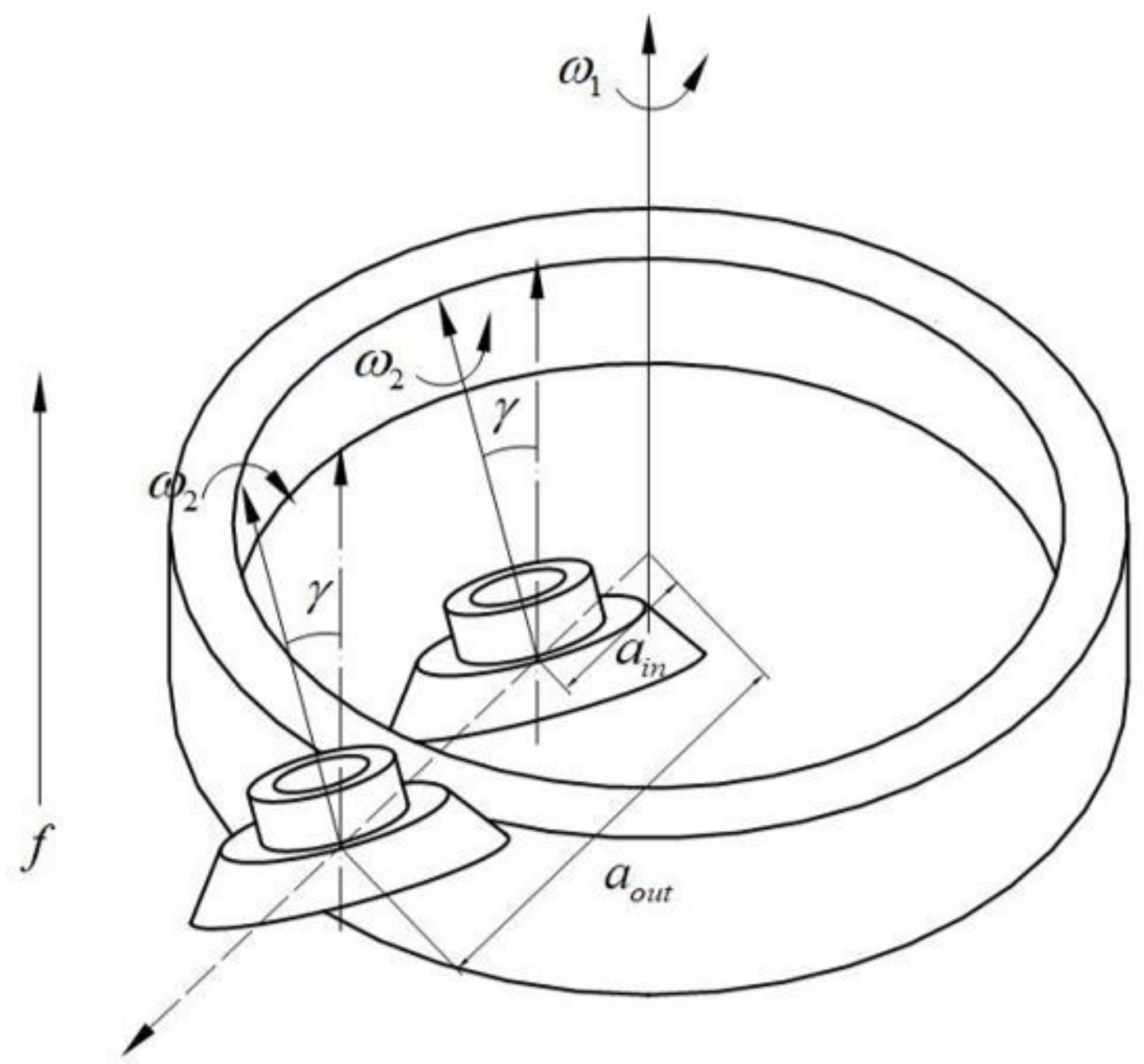

Figure 3

Similarities and differences between the slicing of internal and external gears 


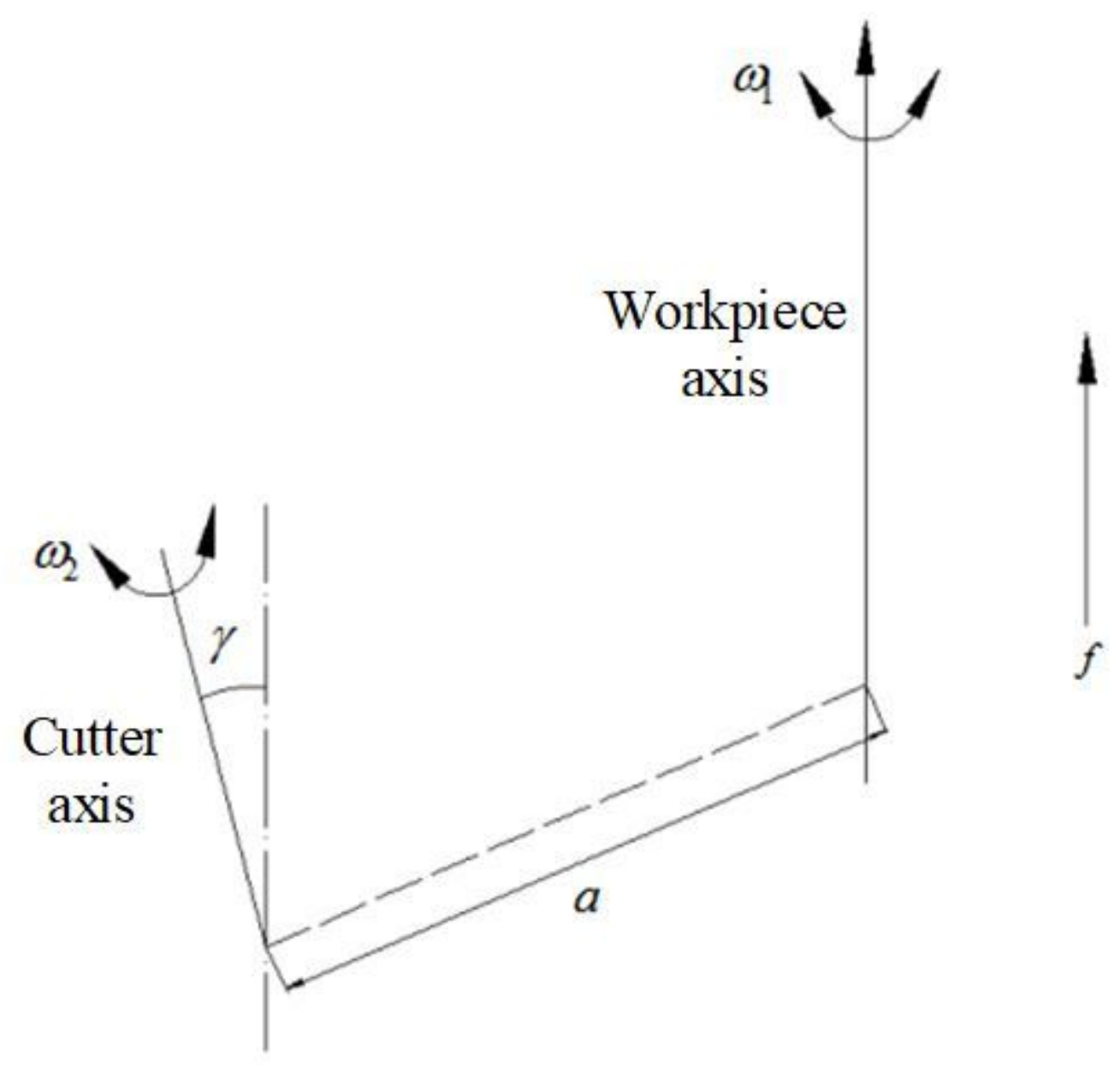

Figure 4

Motion abstract model of gear slicing 


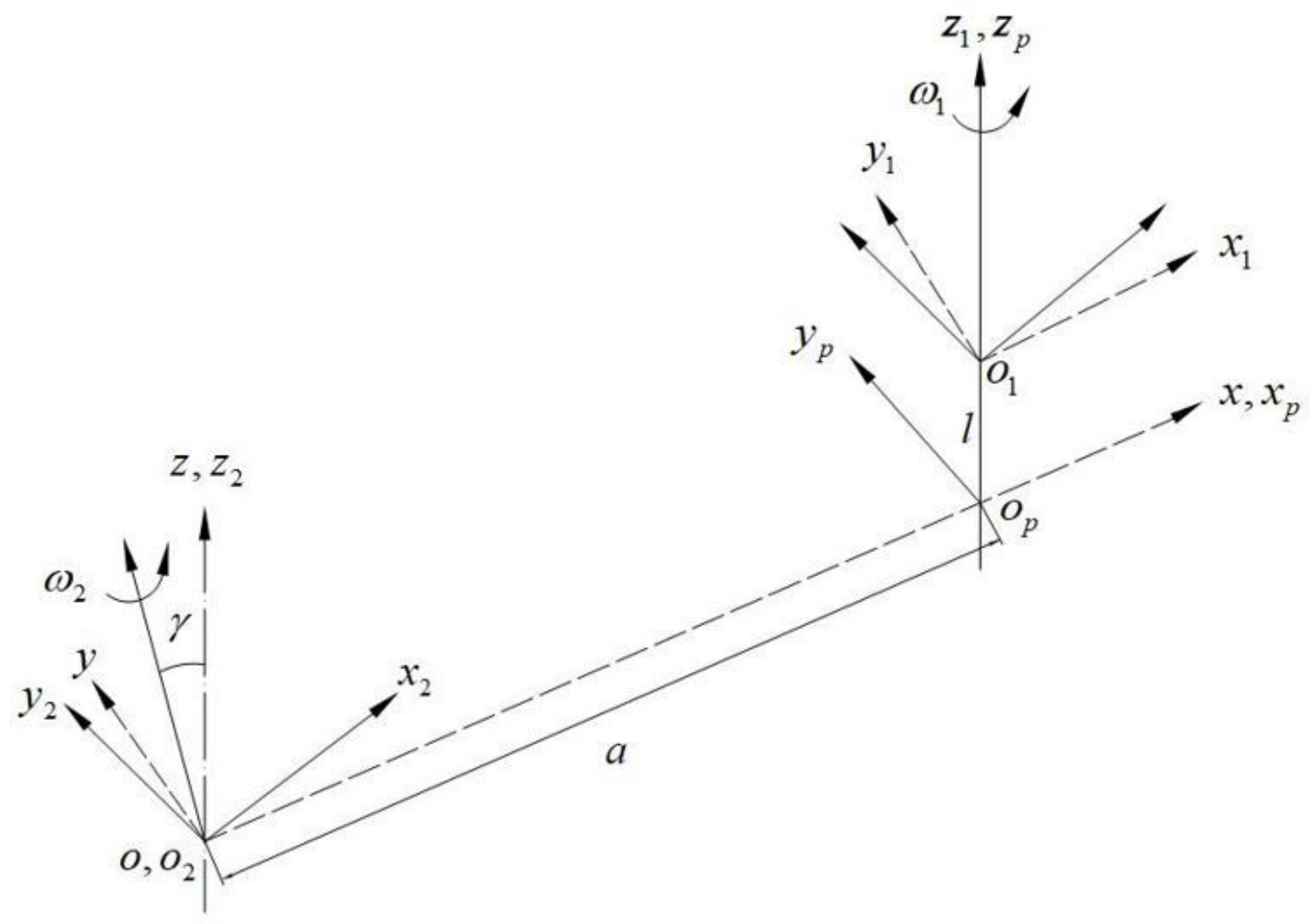

Figure 5

Coordinate system 


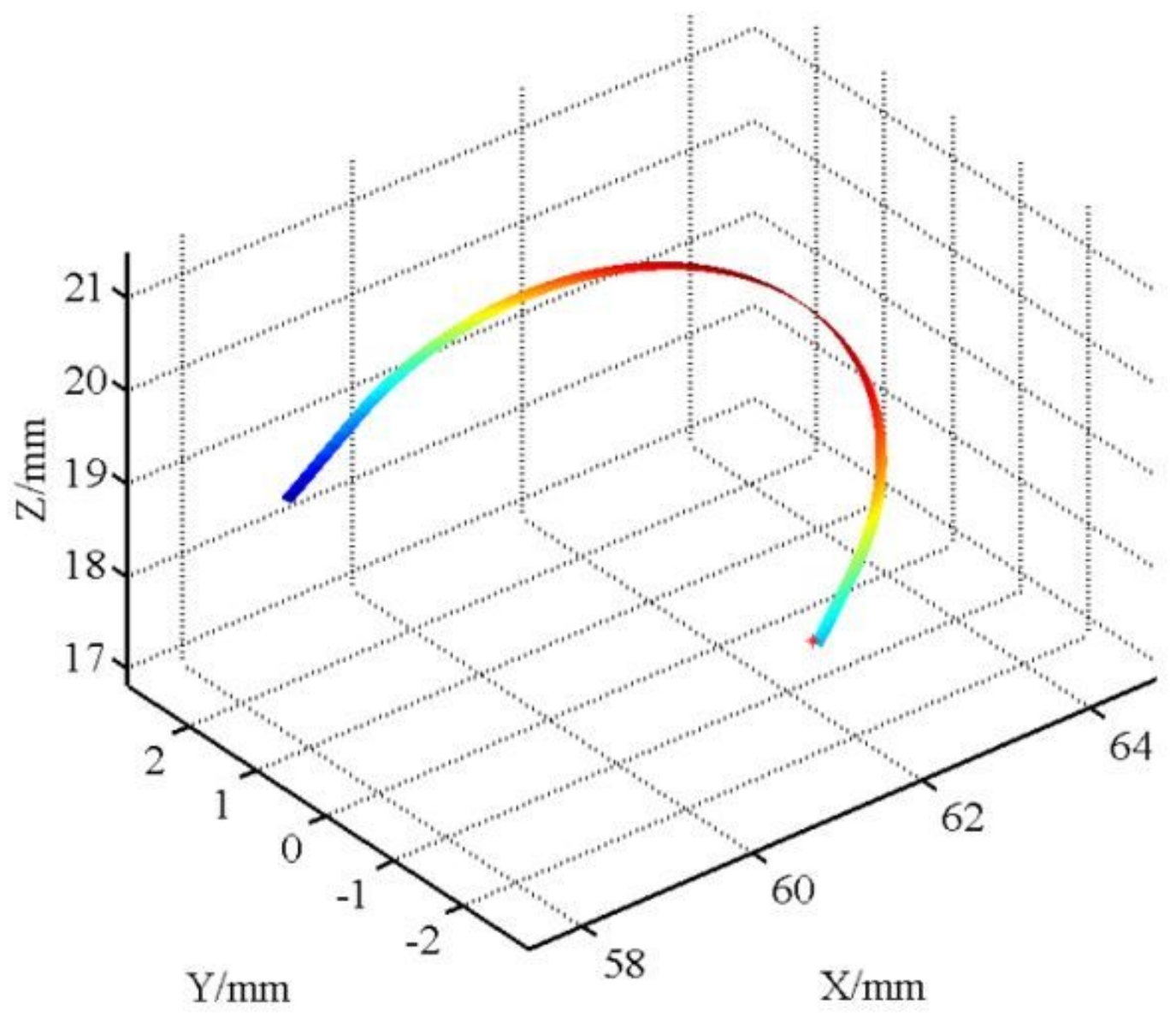

Figure 6

The cutting edge of the ATST-ERA 


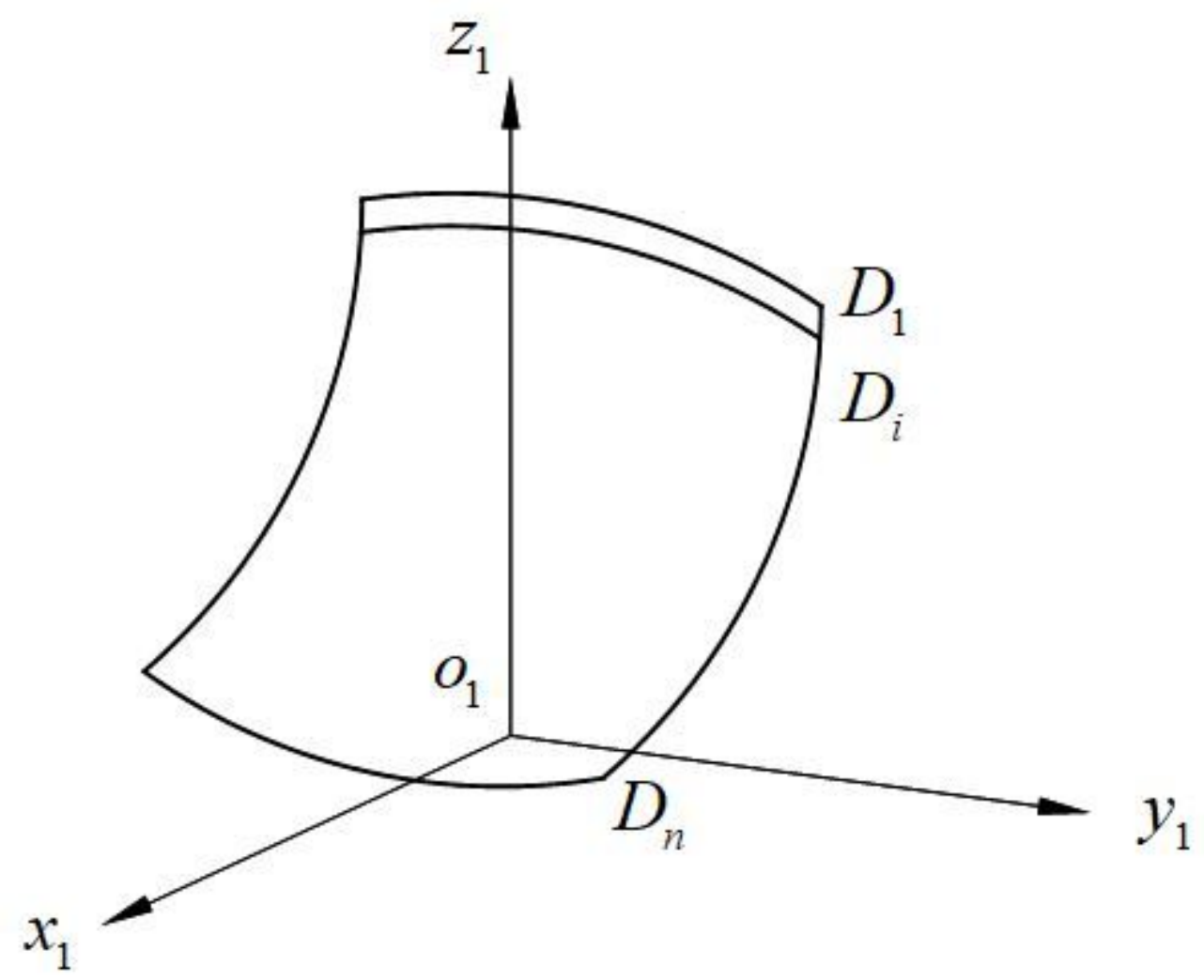

Figure 7

Edge-sweeping surface formed by the cutting edge on the workpiece coordinate system S1 


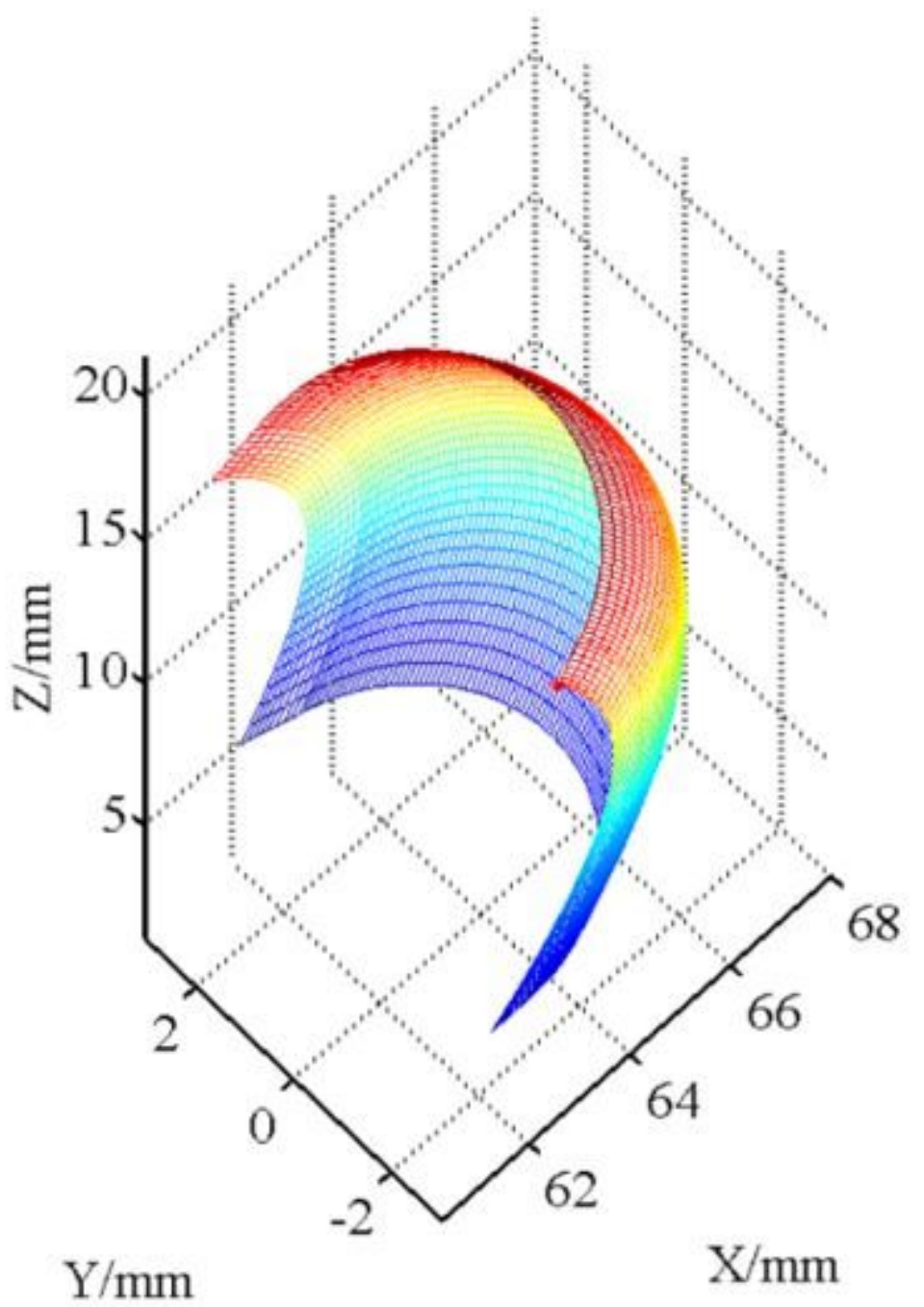

Figure 8

Edge-sweeping surface of the ATST-ERA 


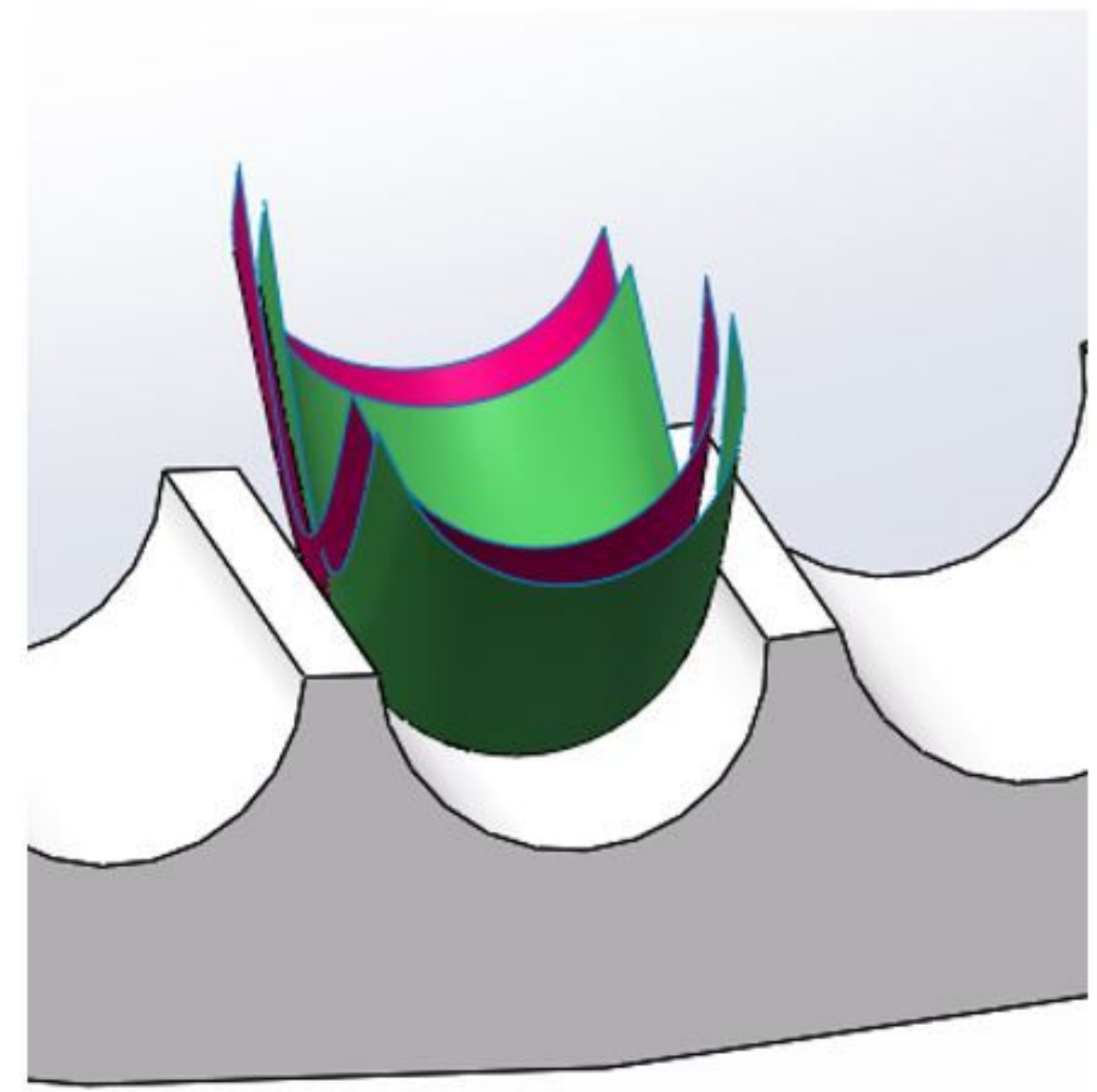

Figure 10

Schematic diagram of the tooth surface of the workpiece swept by the adjacent edge-sweeping surface 


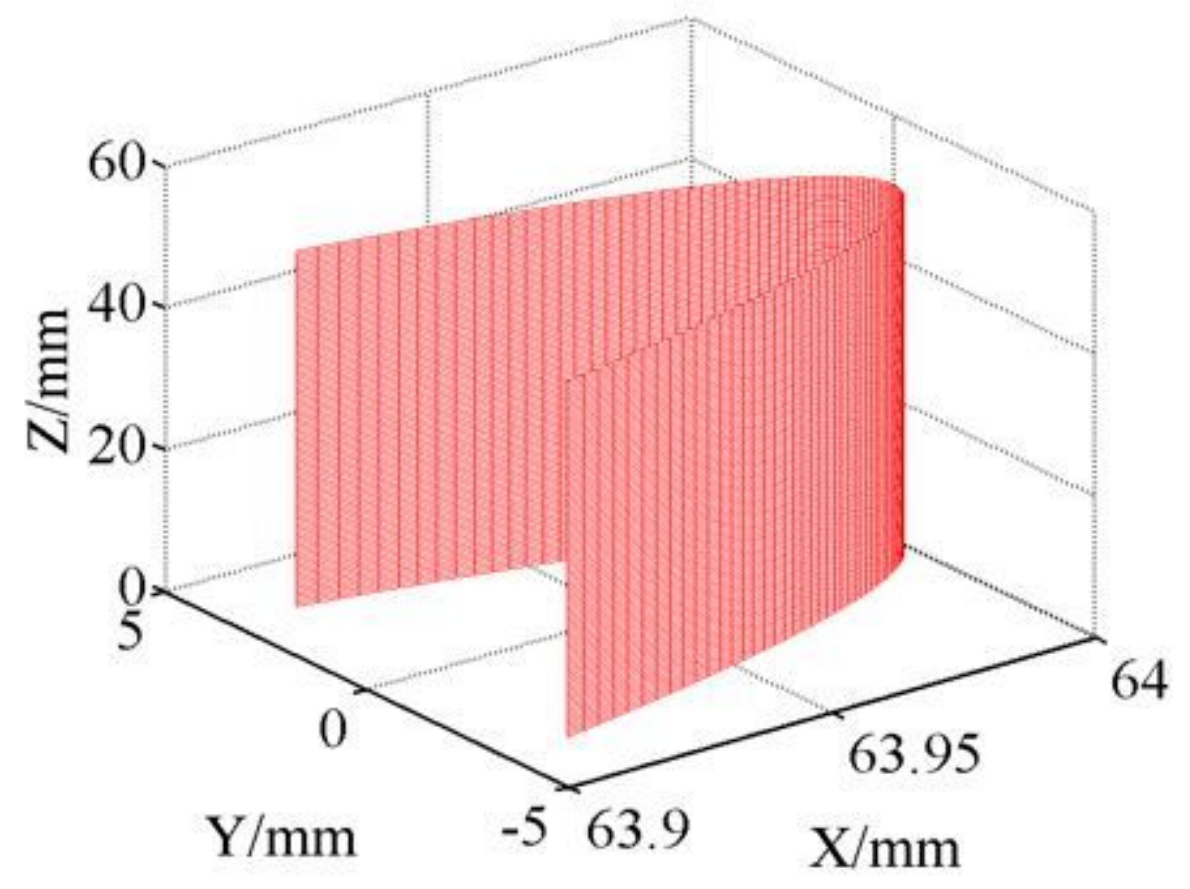

Figure 11

Tooth surface of arc-tooth gear 


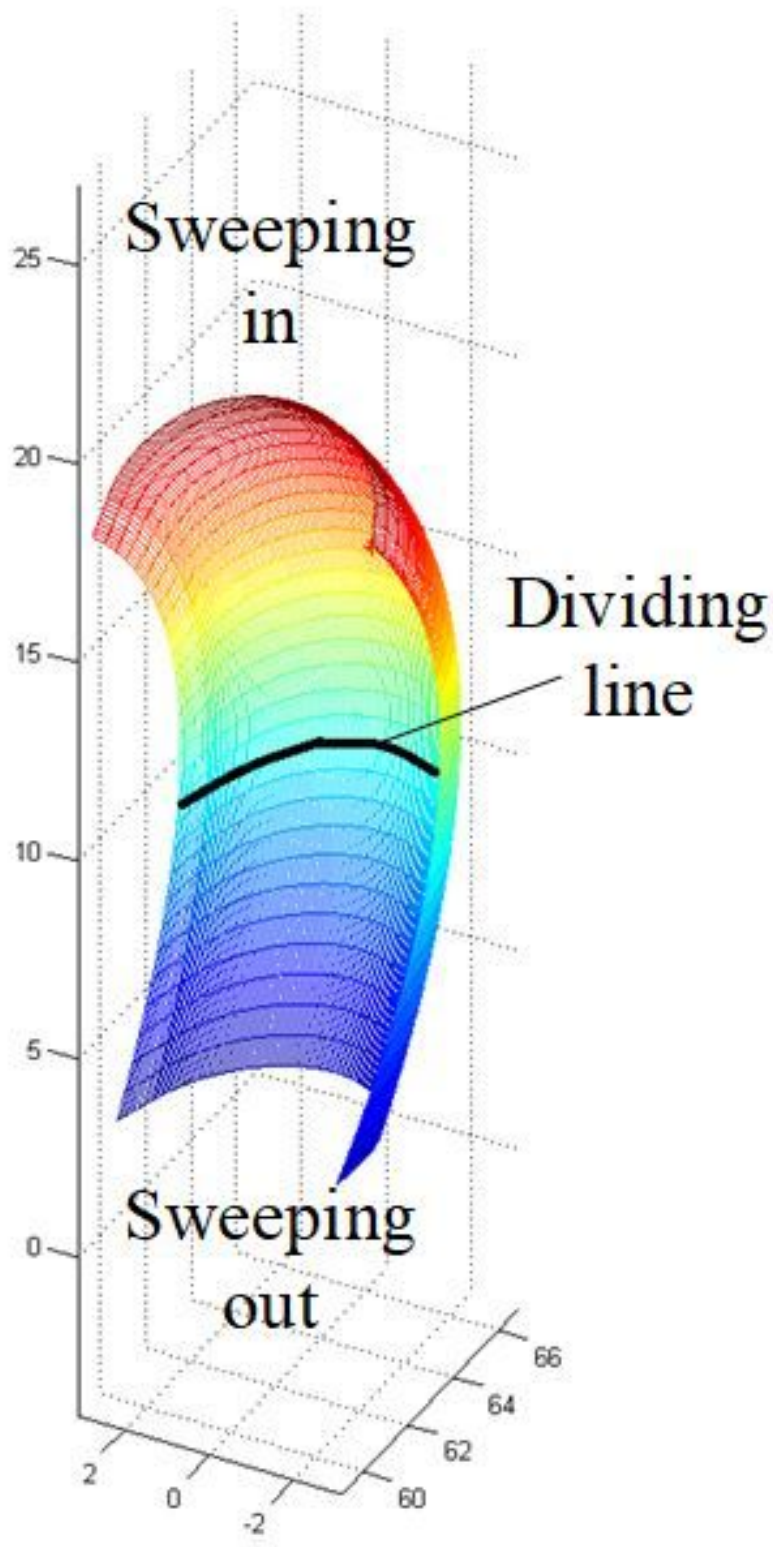

Figure 12

Schematic diagram of division of the edge-sweeping surface 


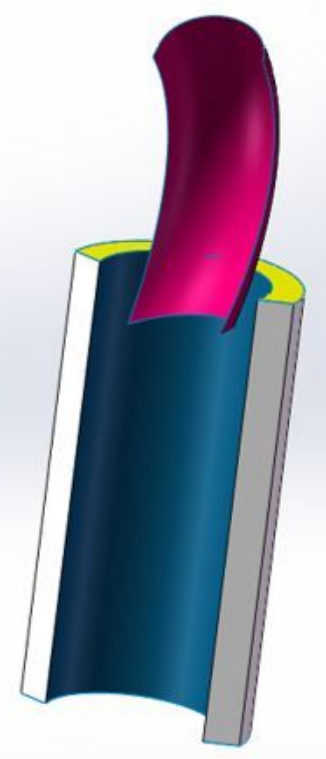

(a)

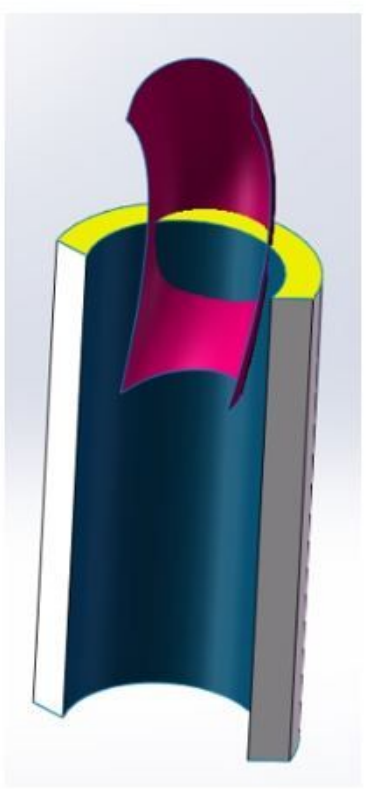

(b)

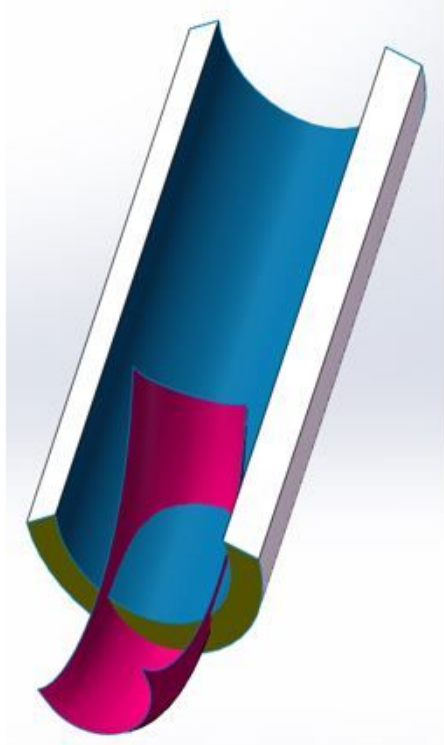

(c)

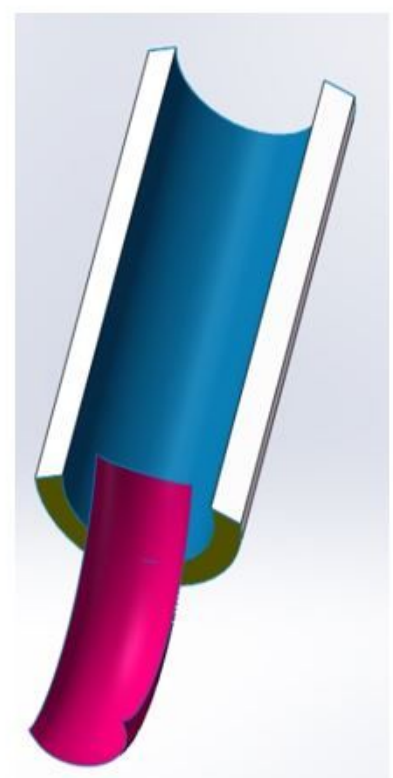

$(d)$

Figure 13

Different cutting states during gear slicing process: (a) the edge-sweeping surface just touching the upper end of the tooth groove; (b) the dividing line of the edge-sweeping surface just touching the upper end of the tooth groove; (c) the dividing line of the edge-sweeping surface just touching the lower end of the tooth groove; (d) the edge-sweeping surface just off the lower end of the tooth groove;

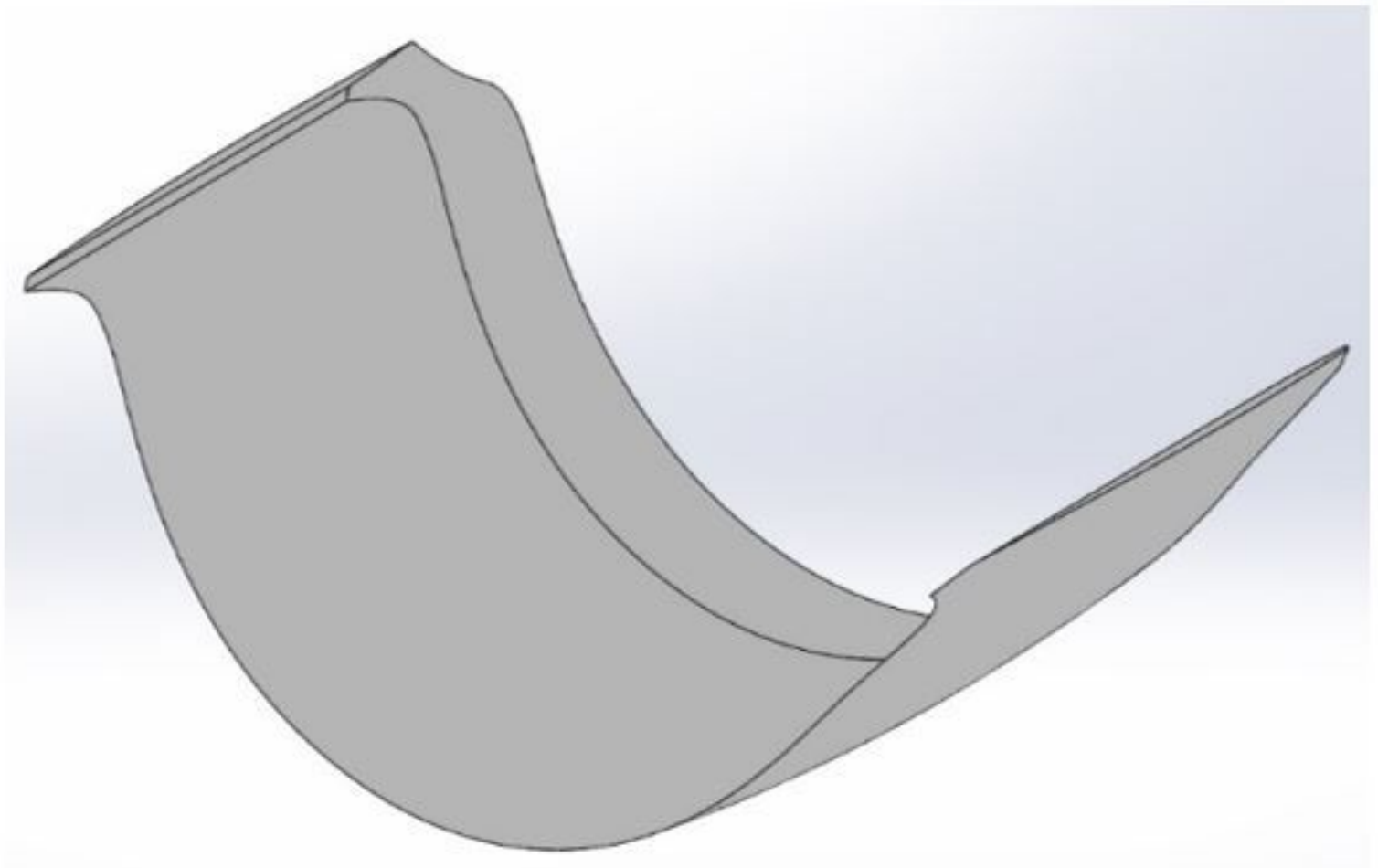


Figure 14

Undeformed chip
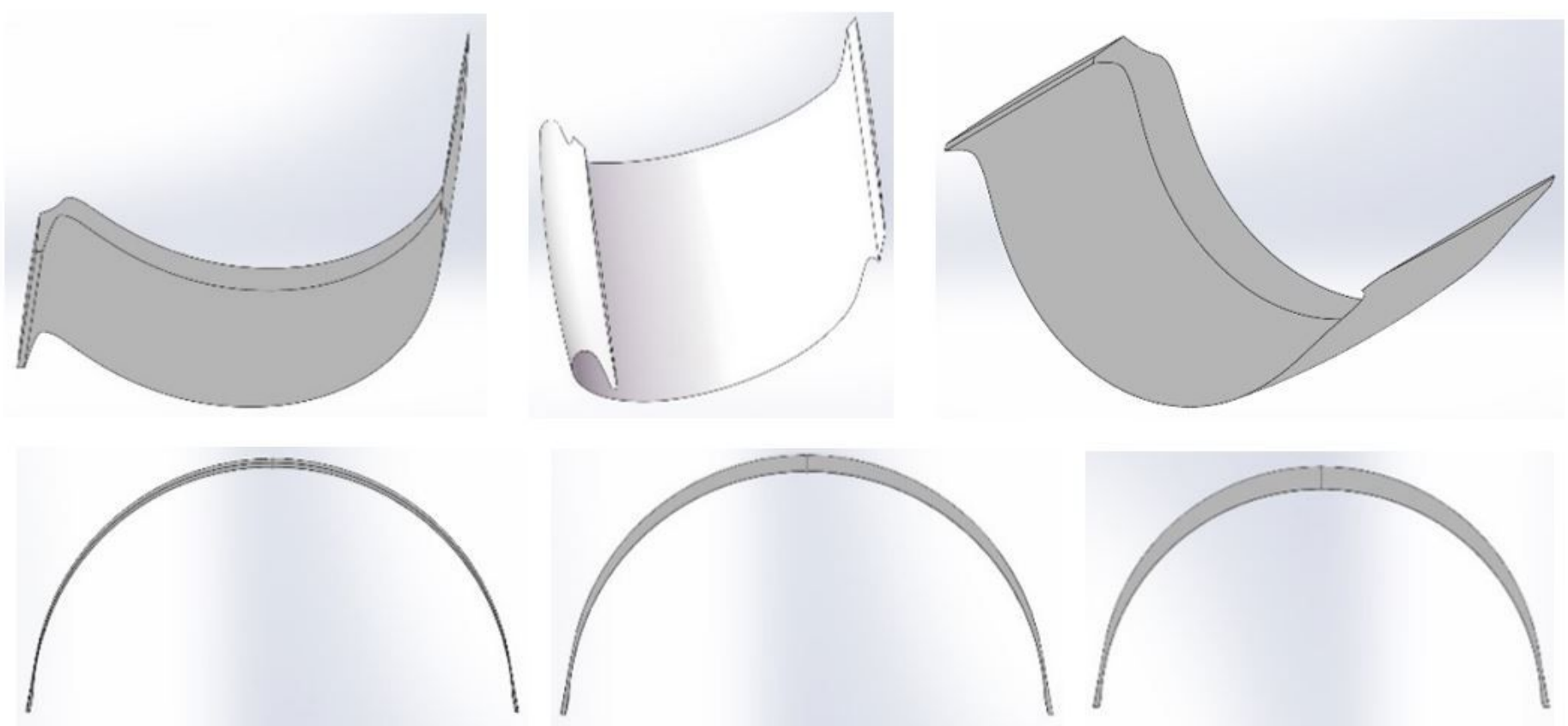

(a)

(b)

(c)

\section{Figure 15}

Undeformed chip obtained by simulation calculation

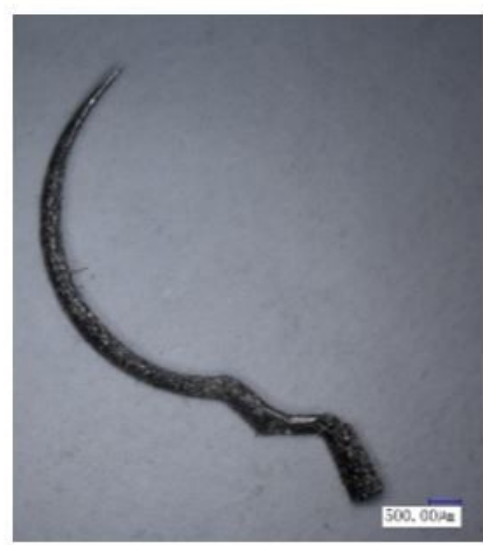

(a)

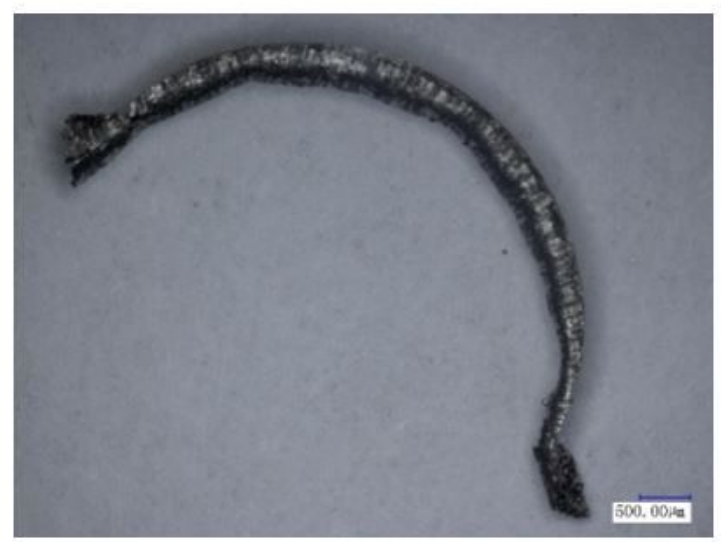

(b)

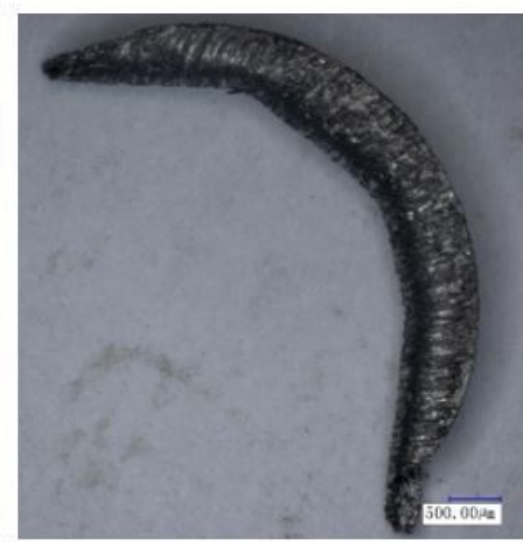

(c)

\section{Figure 16}

The experimentally collected deformed chips 

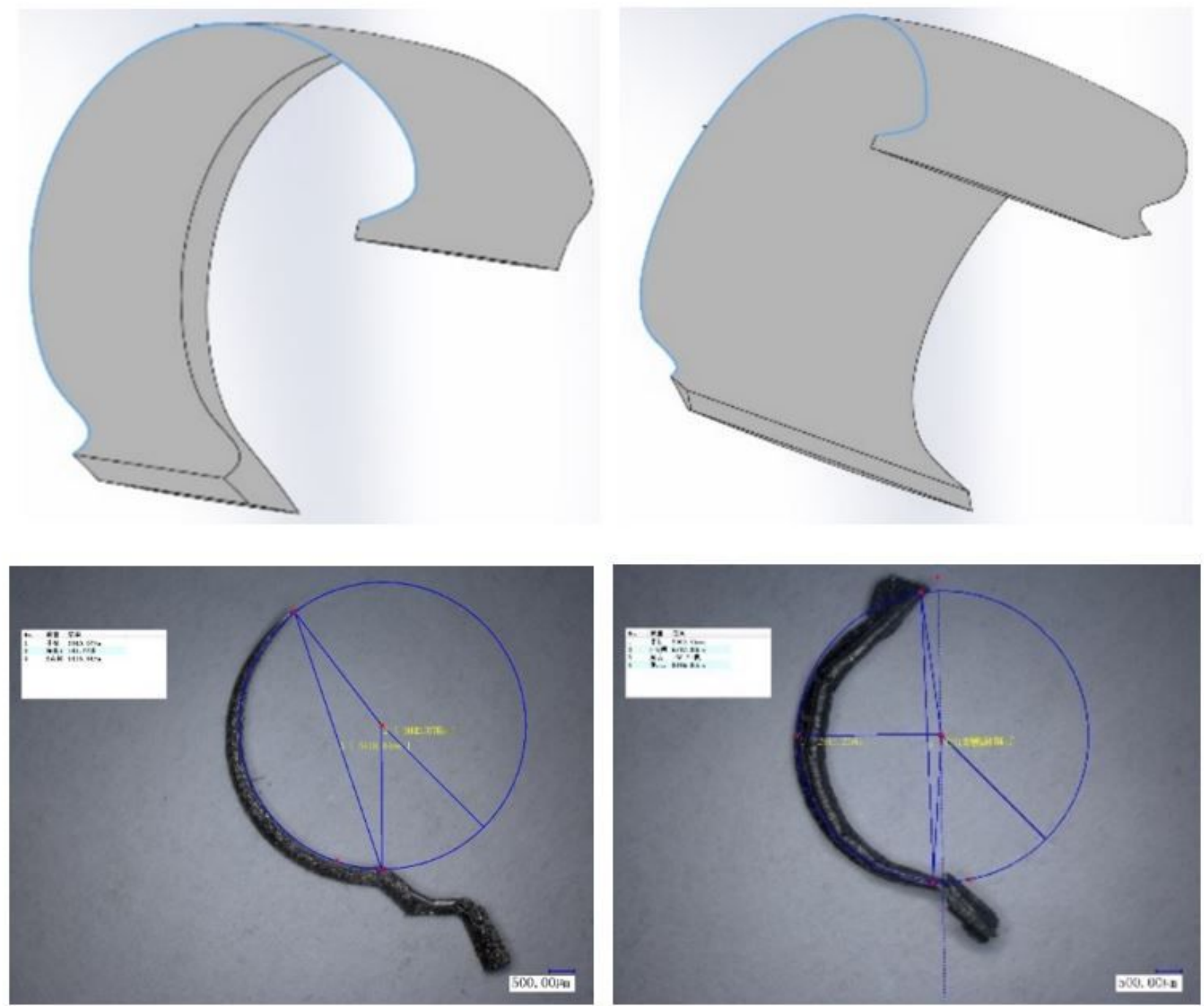

(a)

(b)

\section{Figure 17}

Measurement results of chip shape parameters under different slicing parameters 

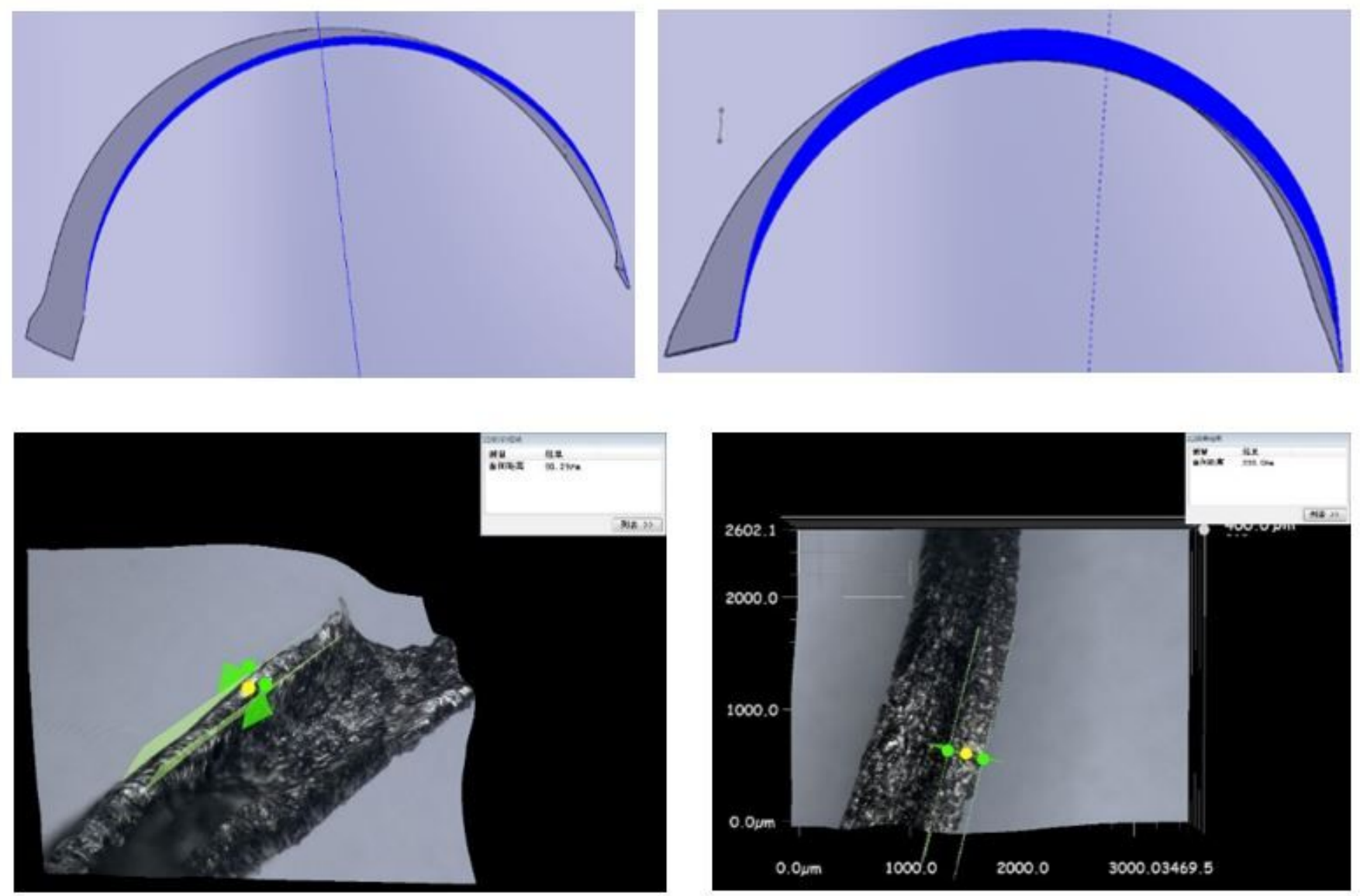

(a)

(b)

\section{Figure 18}

Measurement results of chip thickness under different slicing parameters 


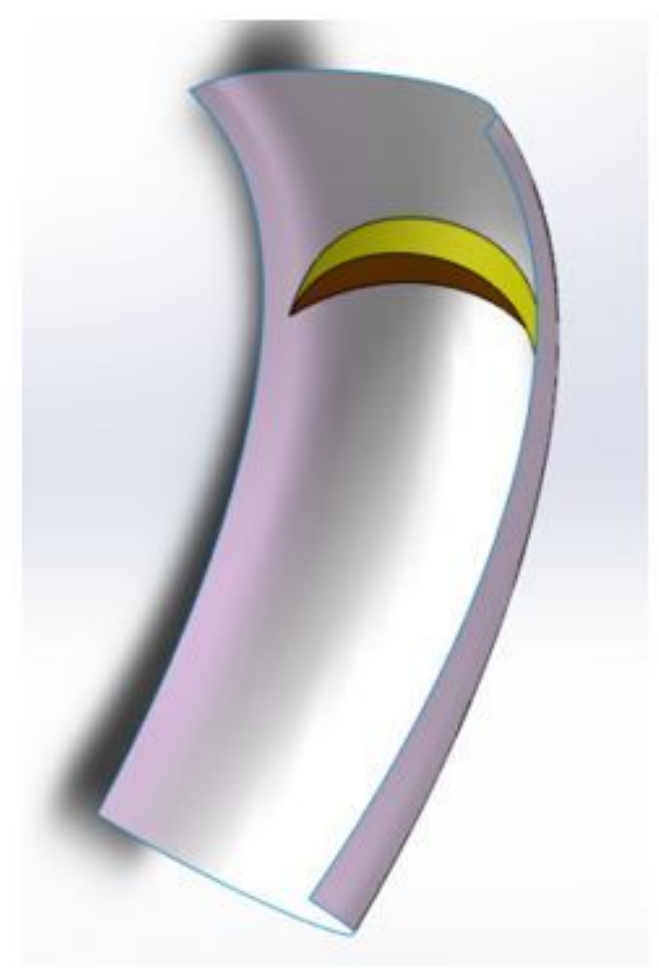

Figure 19

Space sweeping trail of edge-sweeping surface

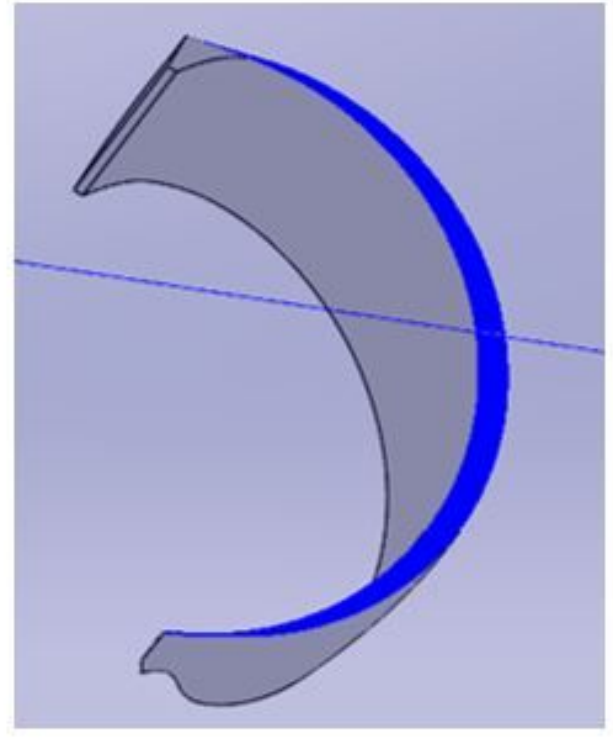

(a)

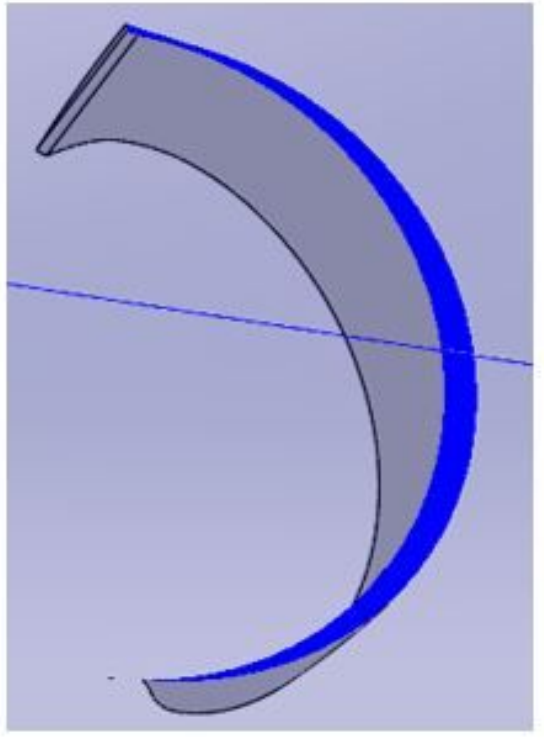

(b)

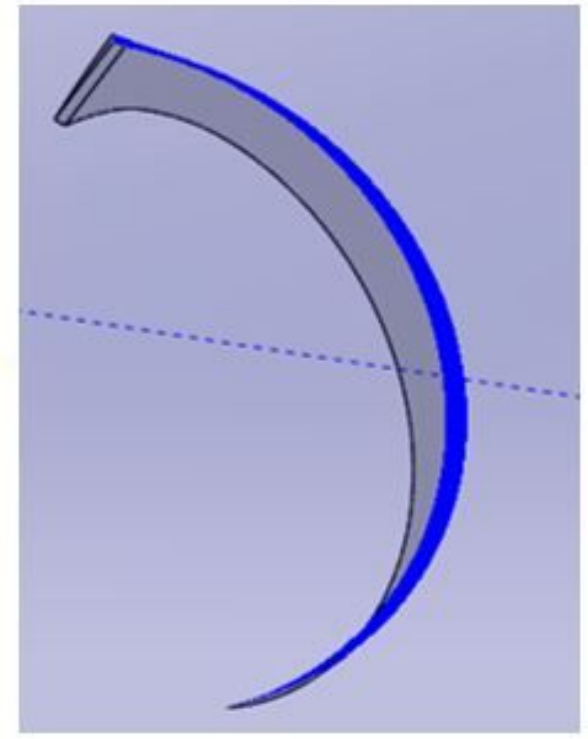

(c)

Figure 20 
Chip thickness corresponding to the cutting edge at different times

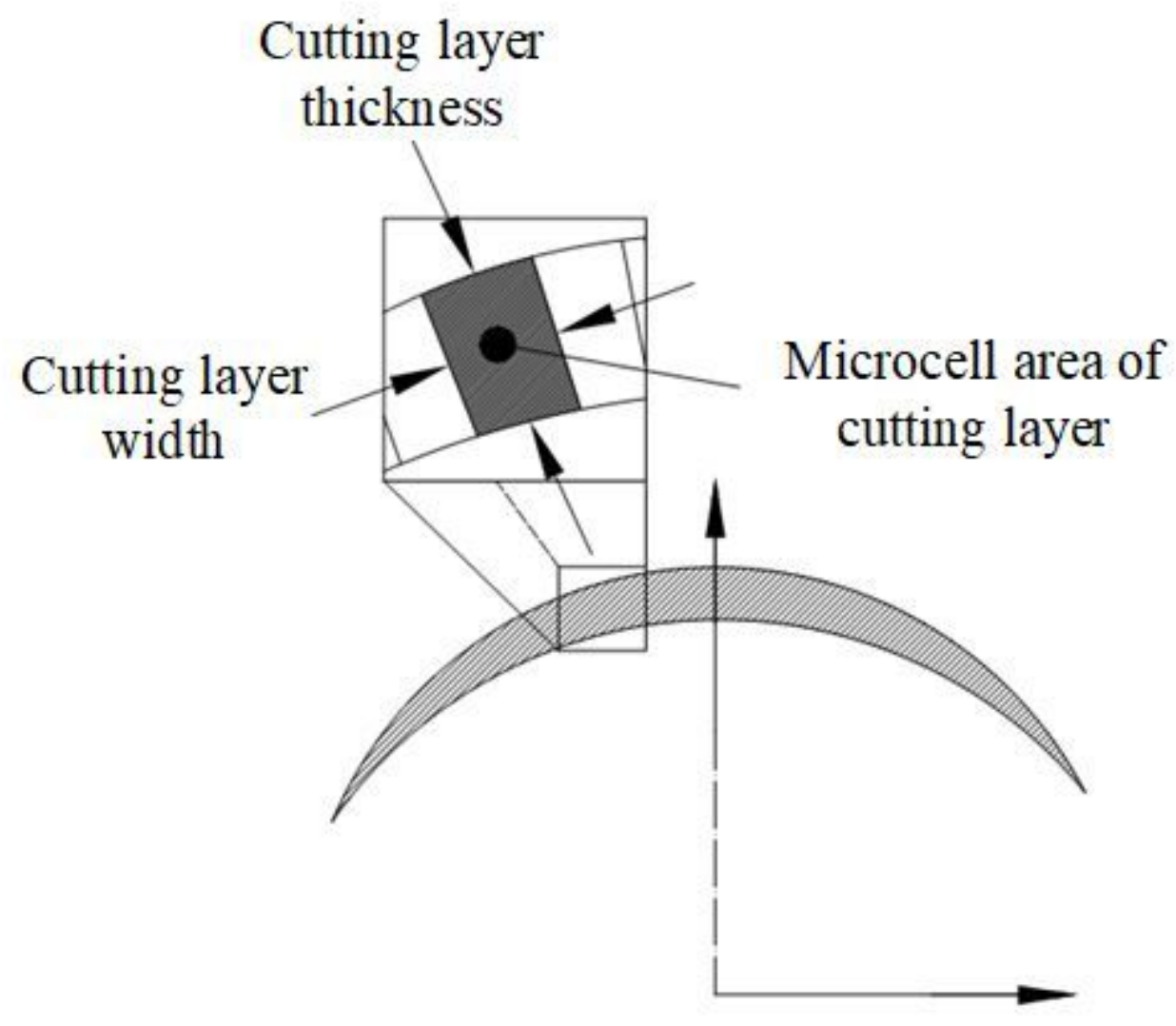

Figure 21

Microcell area at a certain point of the cutting edge

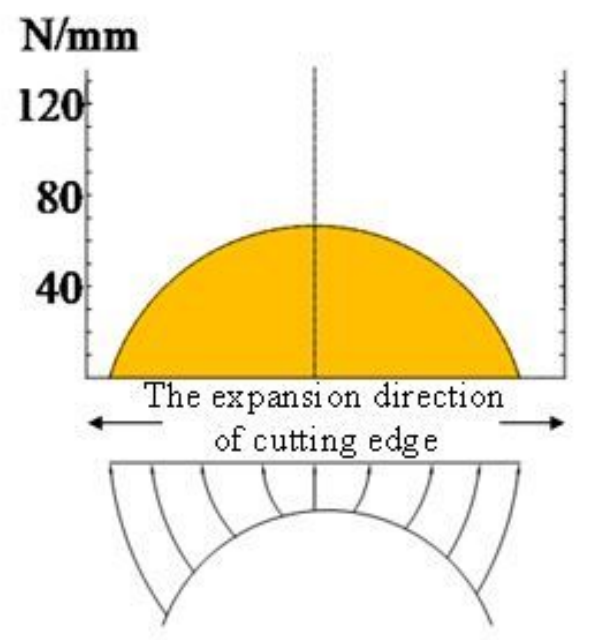

(a) No. 3 cutter teeth
$\mathrm{N} / \mathrm{mm}$

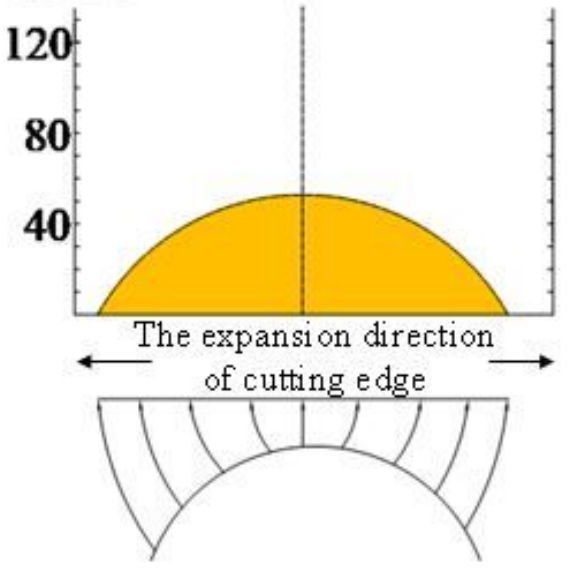

(b) No. 6 cutter teeth
$\mathrm{N} / \mathrm{mm}$

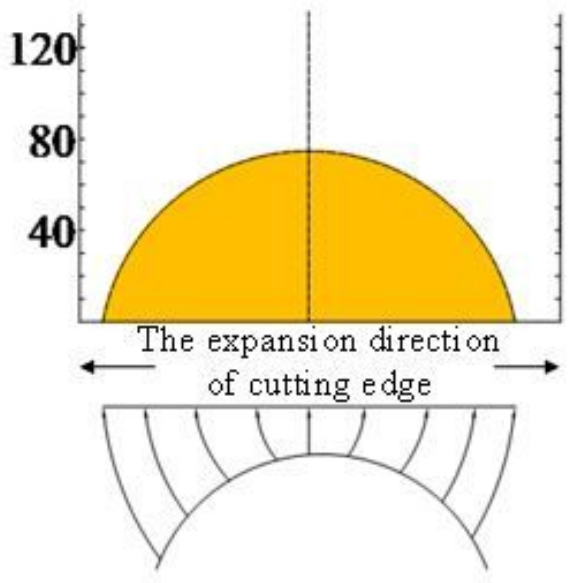

(c) No. 15 cutter teeth 
Load spectrum of No. 3, No. 6 and No. 15 cutter teeth
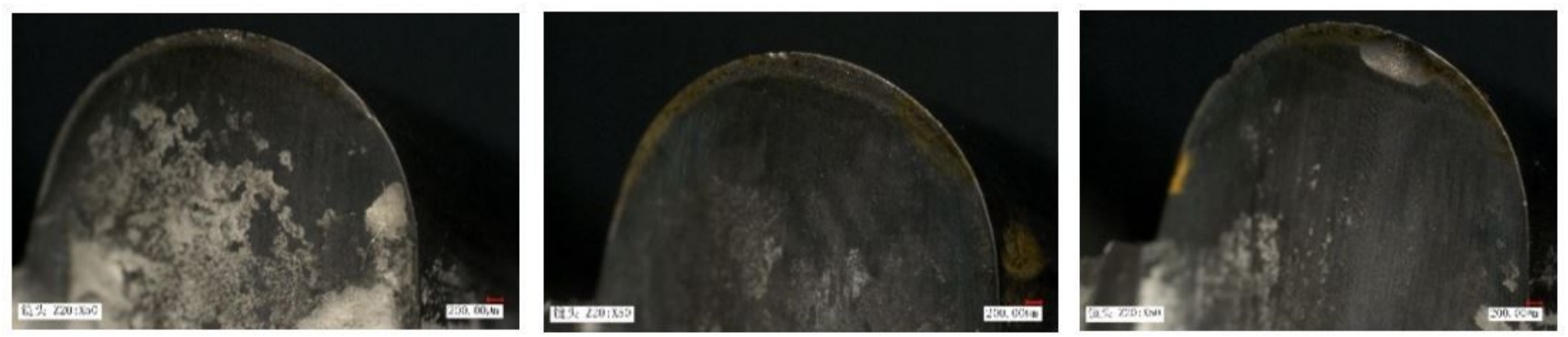

Figure 23

Schematic diagram of wear on rake face of No. 3, No. 6 and No. 15 cutter teeth
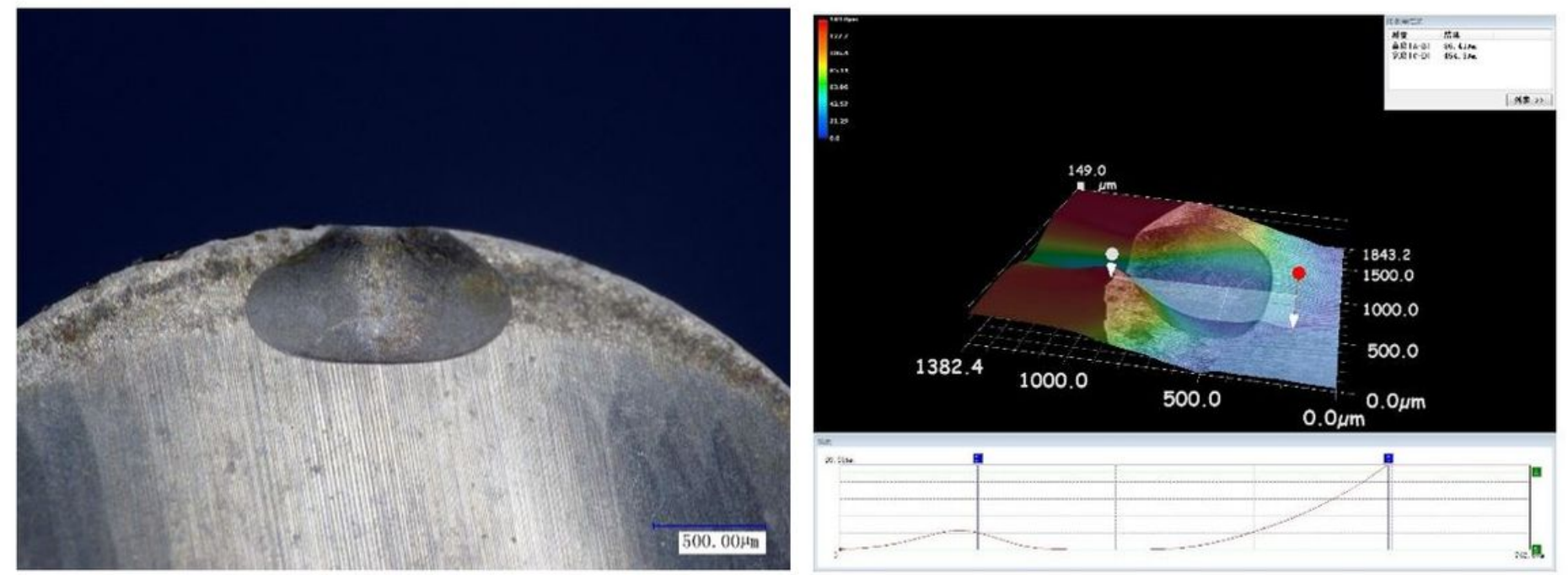

Figure 24

Schematic diagram of crater wear on rake face of No. 15 cutter tooth 


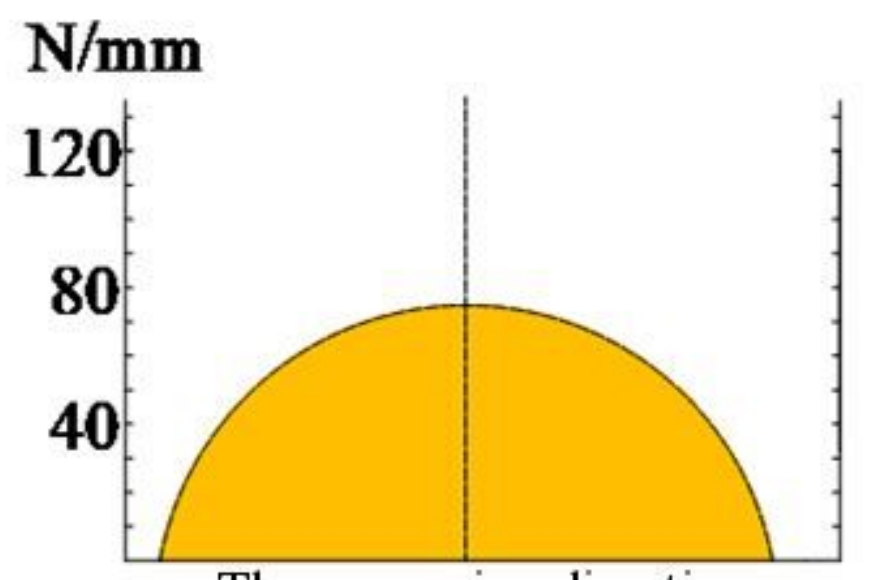

The expansion direction
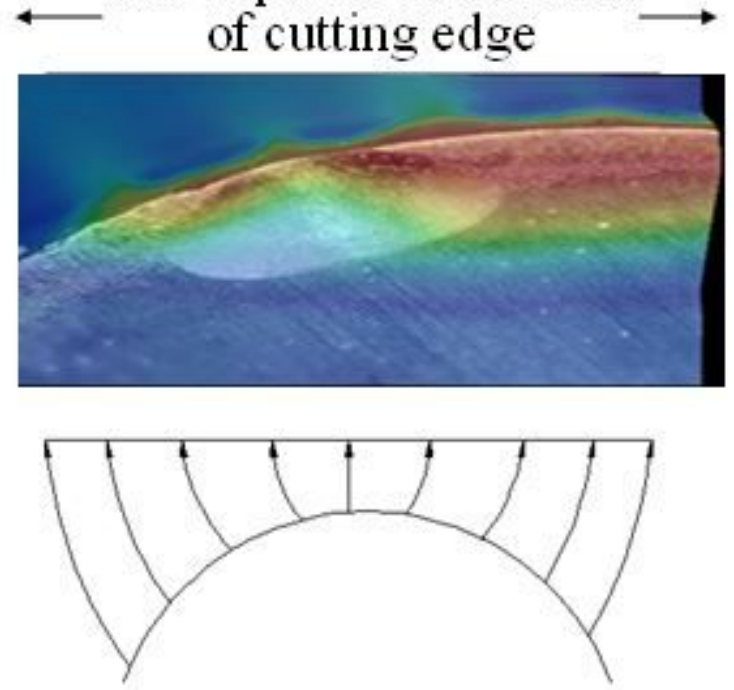

Figure 25

Load spectrum and wear of No. 15 cutter tooth 